\title{
Functional connectivity dynamically evolves on multiple time-scales over static structural connectivity: Models and Mechanisms
}

\author{
Joana Cabral ${ }^{1,2}$, Morten Kringelbach $^{1,2}$ Gustavo Deco $^{4,5}$ \\ 1 Department of Psychiatry, University of Oxford, UK \\ 2 Center for Music in the Brain, Aarhus University, Denmark \\ ${ }^{3}$ Center of Brain and Cognition, Universitat Pompeu Fabra, Barcelona, Spain \\ ${ }^{4}$ ICREA, Institució Catalana de Recerca i Estudis Avancats (ICREA), Spain
}

\begin{abstract}
\end{abstract}
Over the last decade, we have observed a revolution in brain structural and functional Connectomics. On one hand, we have an ever-more detailed characterization of the brain's white matter structural connectome. On the other, we have a repertoire of consistent functional networks that form and dissipate over time during rest. Despite the evident spatial similarities between structural and functional connectivity, understanding how different time-evolving functional networks spontaneously emerge from a single structural network requires analyzing the problem from the perspective of complex network dynamics and dynamical system's theory. In that direction, bottom-up computational models are useful tools to test theoretical scenarios and depict the mechanisms at the genesis of resting-state activity, beyond serving as predictive tools to estimate functional connectivity.

Here, we provide an overview of the different mechanistic scenarios proposed over the last decade via computational models. Importantly, we reinforce the need of implementing additional validation steps in order to refresh the list of candidate scenarios in the light of empirical evidence from MEG-based Envelope Functional Connectivity and Functional Connectivity Dynamics. 


\section{Contents}

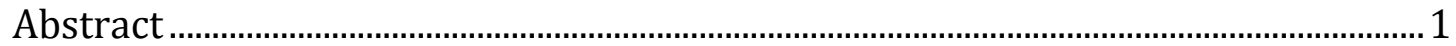

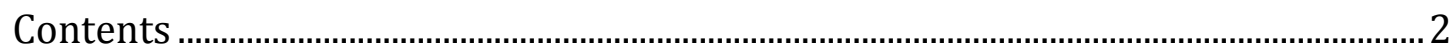

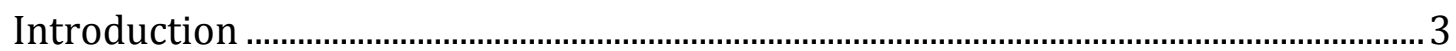

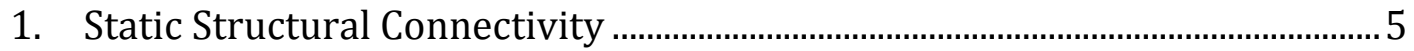

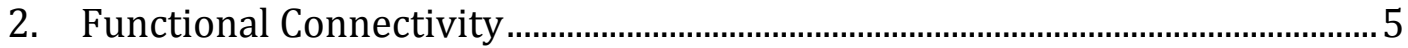

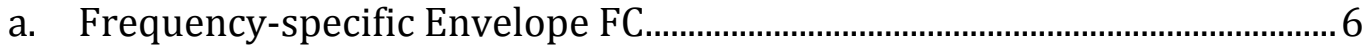

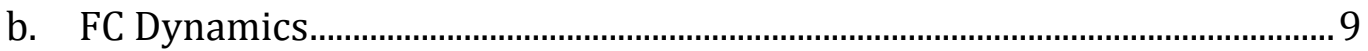

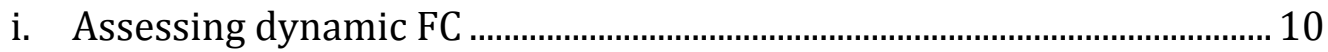

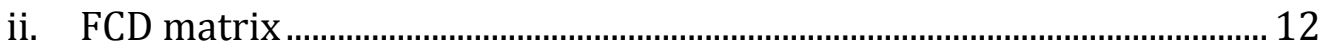

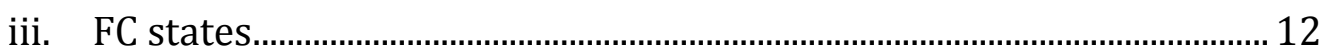

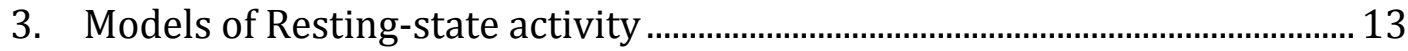

a. Generative mechanistic scenarios ……....................................................... 14

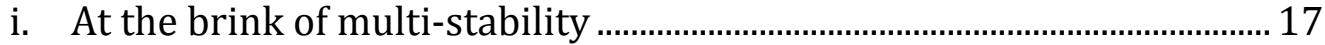

ii. Intermittent self-organization from chaos ................................................ 18

iii. In the vicinity of a supercritical bifurcation............................................. 18

iv. Meta-stable Chimera Synchronization ...................................................... 21

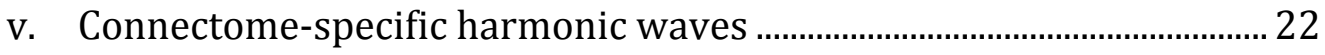

vi. Noise deviations around the equilibrium ................................................. 23

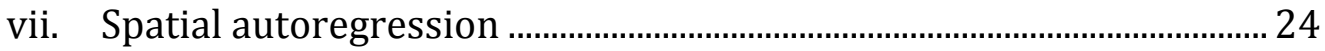

b. Validation of Resting-state models ……..................................................... 24

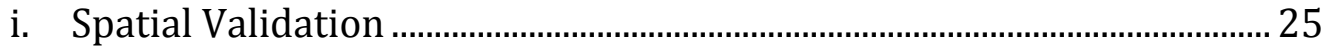

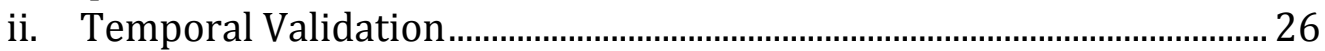

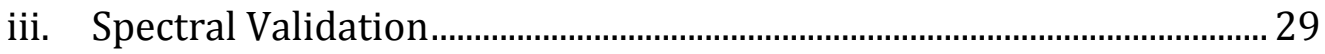

c. A new generation of resting-state models..................................................... 30

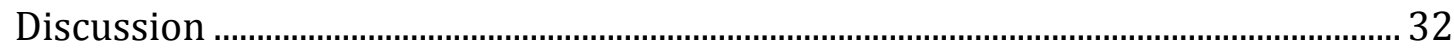

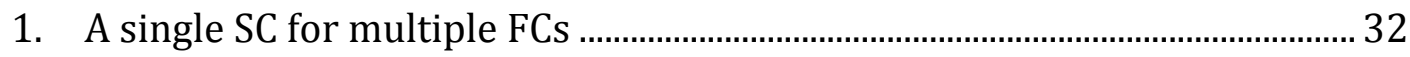

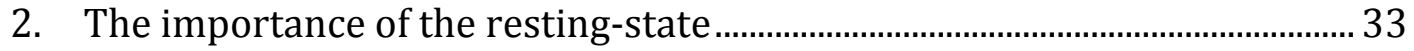

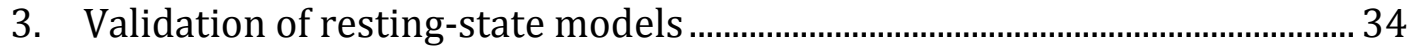

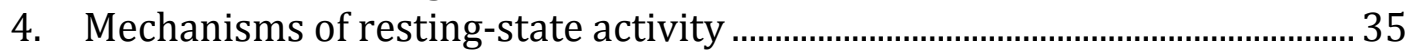

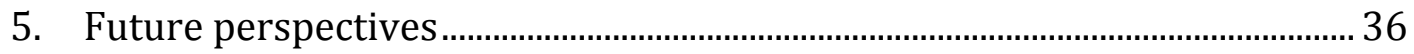

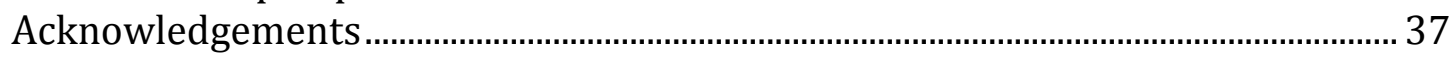

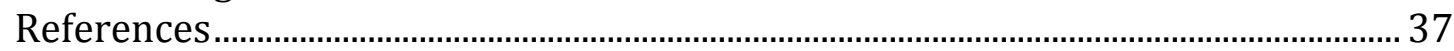




\section{Introduction}

Within the rapidly growing field of brain Connectomics, two main modes of connectivity emerge: Structural and Functional (Biswal et al., 1995, Sporns et al., 2005, Hagmann et al., 2008, Sporns, 2011). While the first refers to the anatomical white-matter fibers connecting brain areas, which remain relatively constant over short time scales, the latter refers to any measure of co-variation between brain signals at different locations and depends strongly on the paradigm and the time-window considered (Figure 1).

\section{CONNECTIVITY}

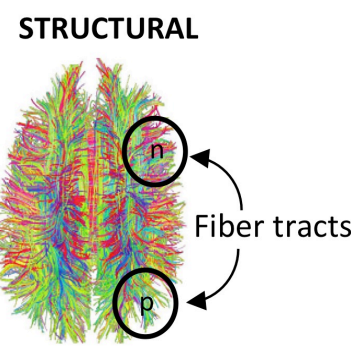

FUNCTIONAL
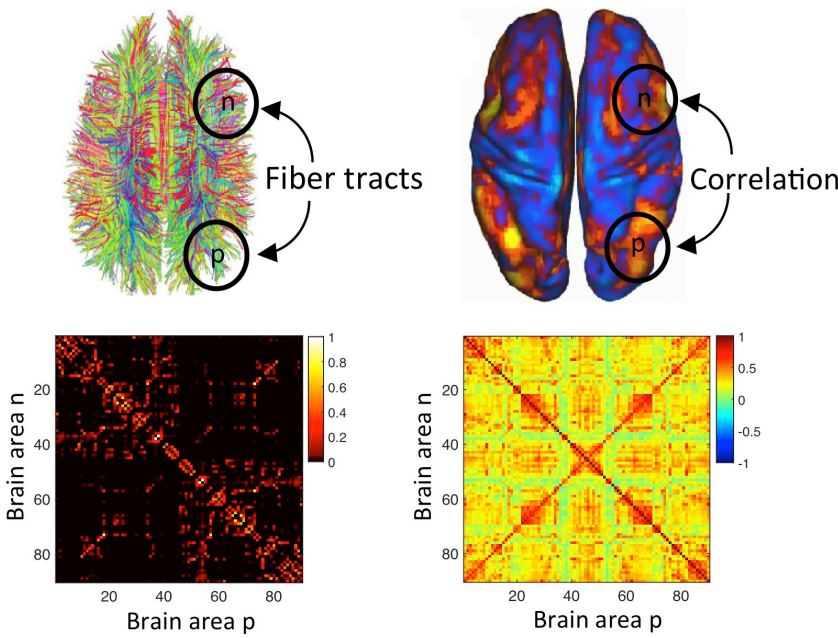

Figure 1 - Structural vs functional connectivity. (Left) Advanced tractography algorithms allow reconstructing the white matter fiber tracts from Diffusion-MRI. The structural connectivity matrix SC(n,p) is estimated in proportion to the number of fiber tracts detected between any two brain areas $n$ and $p$. (Right) On the other hand, the functional connectivity matrix $\mathrm{FC}(\mathrm{n}, \mathrm{p})$ is computed as the correlation between the brain activity (e.g. BOLD signal) estimated in areas $\mathrm{n}$ and $\mathrm{p}$ over the whole recording time. Here, the areas refer to 90 non-cerebellar brain areas from the AAL template.

When computed over sufficiently long sessions at rest, the whole-brain map of functional connections, generally termed Functional Connectome, reveals some similarities with the Structural Connectome (Greicius et al., 2009, van den Heuvel et al., 2009, Biswal et al., 2010, Hermundstad et al., 2013). However, this 
relationship is not trivial and has been subject of investigation from several theoretical and computational research groups over the last decade (Honey et al., 2007, Ghosh et al., 2008b, Izhikevich and Edelman, 2008, Deco et al., 2009, Honey et al., 2009, Cabral et al., 2011, Deco and Jirsa, 2012, Deco et al., 2013, Cabral et al., 2014a, Messe et al., 2015, Ponce-Alvarez et al., 2015, Atasoy et al., 2016, Robinson et al., 2016, Spiegler et al., 2016). Following different reduction lines and alternative mechanistic approaches (which we will review below), these works have demonstrated via whole-brain network models how the neuroanatomical network structure can shape spontaneous brain activity on very slow time-scales, giving rise to consistent patterns of Functional Connectivity (FC).

Given the wide variety of candidate scenarios to explain the source of restingstate FC patterns, it becomes necessary to establish further model constrains in order to refresh the list of candidate scenarios and foster our understanding of the network mechanisms underlying resting-state activity. Novel insights into resting-state FC have been revealed by MEG studies (de Pasquale et al., 2010, Brookes et al., 2011b, Hipp et al., 2012, Hipp and Siegel, 2015) and by investigations on the temporal dynamics of FC (Chang and Glover, 2010, Handwerker et al., 2012, Smith et al., 2012, Hutchison et al., 2013a, Allen et al., 2014, Zalesky et al., 2014).

In the present work, we provide an overview of the latest findings in the literature of resting-state $\mathrm{FC}$, focusing on the properties observed at finer temporal scales with MEG and the dynamical properties of FC. Then, we review existing whole-brain generative models of resting-state activity, with a special focus on their mechanistic scenarios. Finally, we compare the different candidate 
models and comment on their capacity to survive the constrains imposed by novel empirical evidence.

\section{Static Structural Connectivity}

The development of advanced tractography algorithms applied to Diffusion-MRI has allowed detecting - non-invasively and at the whole-brain level - the white matter fiber tracts connecting brain areas (Sporns et al., 2005, Hagmann et al., 2008). This permits the construction of large-scale networks where nodes represent brain areas and links reflect the anatomical connectivity between them. Considering macro-scale brain parcellations in the order of 100 regions, the Structural Connectome of healthy adults remains relatively stable across sessions when compared with the rapid changes occurring at the dynamical level (Sporns, 2011). Any alterations occurring at this macro-scale are typically very slow, either when associated to the brain's natural development and aging, or to disease (Bartzokis et al., 2003, Hagmann et al., 2010, Takeuchi et al., 2010). Note however, that the entire map of neural connections ambitioned by the Human Connectome Project (www.humanconnectomeproject.org) may reveal higher individual variability and changes on shorter time-scales. Yet, it remains acceptable to consider that the Structural Connectivity (SC) is static when compared to the fast changes observed in FC.

\section{Functional Connectivity}

Functional Connectivity is defined as the temporal dependence of neuronal activity patterns of anatomically separated brain regions typically measured as the co-variation between brain signals originating at different locations (Aertsen et al., 1989, Friston et al., 1993, van den Heuvel and Hulshoff Pol, 2010). The 
term Functional Connectome is generally used to refer to the whole map of functional connections across the whole brain (Biswal et al., 2010). Since meaningful large-scale functional networks have been detected from correlations between slow $(<0.1 \mathrm{~Hz})$ BOLD signal fluctuations at rest (Biswal et al., 1995, Lowe et al., 1998, Damoiseaux et al., 2006), resting-state fMRI has been the leading technique to measure brain-wide FC (Biswal et al., 2010, van den Heuvel and Hulshoff Pol, 2010). Assuming a stationary perspective, correlations are commonly measured over the whole recording time, resulting in a single, temporally invariant Functional Connectome, which can be expressed in mathematical terms as a grand-average FC matrix, where each entry $F C(n, p)$ refers to the correlation between the signals averaged over all voxels in brain areas $n$ and $p$. This approach allows for a direct comparison with the Structural Connectome defined over the same parcellation scheme (van den Heuvel et al., 2009) and has been particularly useful for the optimization and validation of resting-state models through the comparison with the simulated FC matrix (Honey et al., 2009, Cabral et al., 2011, Deco and Jirsa, 2012, Deco et al., 2013, Messe et al., 2015, Ponce-Alvarez et al., 2015).

\section{a. Frequency-specific Envelope FC}

Although BOLD signal correlations have proved meaningful in the study of resting-state FC (Biswal et al., 2010, van den Heuvel and Hulshoff Pol, 2010), an important shortcoming of fMRI approaches is that fluctuations occurring on the faster timescales of neurophysiological processes $(\sim 2-100 \mathrm{~Hz})$ are not captured (Engel et al., 2013). For this reason, the fast local dynamics has been left largely unconstrained in models of resting-state activity (Honey et al., 2009, Deco et al., 
2011, Deco et al., 2013, Cabral et al., 2014a). Exploring the neurophysiological counterpart of BOLD signal activations, experimental studies have shown a relationship with local increases in the power of neural activity, not only in the gamma-frequency band (Logothetis et al., 2001, Leopold et al., 2003, Nir et al., 2007), but also at lower frequencies (He et al., 2008, Scholvinck et al., 2010, Magri et al., 2012, Tagliazucchi et al., 2012b, Keller et al., 2013). However, for accurately measuring resting-state FC non-invasively and at the whole-brain level using MEG, the correlation patterns resulting from volume conduction need to be suppressed before analyzing functional connectivity. Such methods have only recently become available bringing insightful information for the investigation of resting-state FC occurring at faster time-scales (Brookes et al., 2011a, Brookes et al., 2011b, Hipp et al., 2012, Engel et al., 2013, Marzetti et al., 2013, Hipp and Siegel, 2015). These studies point in the direction that the BOLD signal fluctuations observed in the brain at rest are associated to aperiodic fluctuations in the power of neural oscillations occurring in a particular frequency range (see Figure 2). Since the information is assumed to be carried in the power (or squared amplitude) of the oscillations in a given frequency band, these oscillations are generally termed carrier oscillations. 


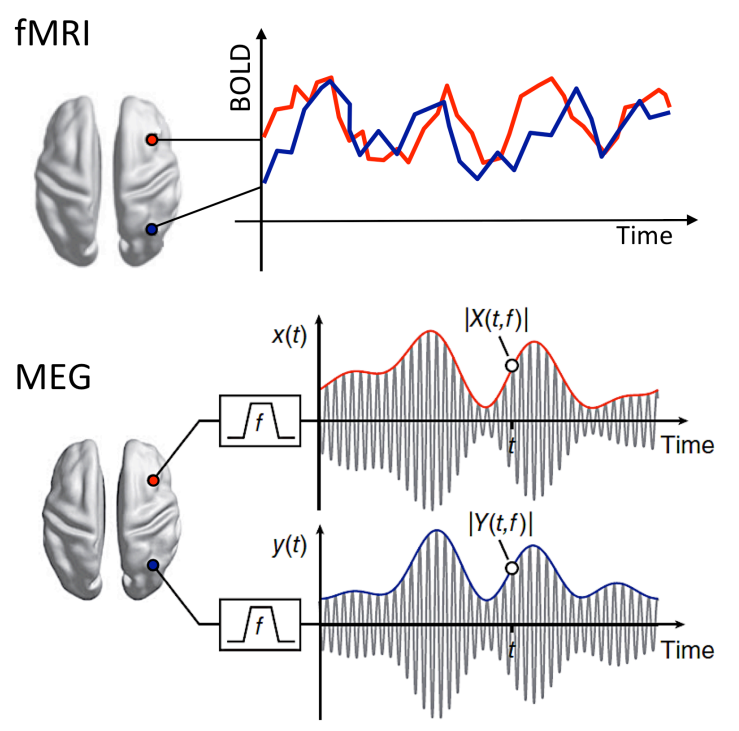

Figure 2 - Functional Connectivity assessed with fMRI or MEG. (Top) fMRI-based functional connectivity is assessed as the correlation between the BOLD signals recorded at different locations. (Bottom) Resting-state functional connectivity in MEG is assessed by first band-pass filtering the MEG signal (before or after the source estimation), extracting the amplitude envelope (red and blue lines) of the underlying carrier oscillation (black) and subsequently computing the correlation between envelopes. This strategy results in a set of frequency-specific FC maps.

According to Brookes et al. (2011b) and Hipp et al. (2012), the best agreement between MEG-based and fMRI-based FC is obtained for carrier oscillations in the alpha and beta frequency ranges $(8-32 \mathrm{~Hz})$. However, a wider range of frequencies between 2 to $128 \mathrm{~Hz}$ has been found to play a role in shaping the large-scale dynamical interactions yielding functional networks (Hipp and Siegel, 2015). Notably, different fMRI-based functional connections were associated to different carrier frequencies, showing that BOLD correlation reflects different types of neuronal interactions across the brain (Hipp and Siegel, 2015).

In order to consider the FC occurring at different carrier frequencies, one approach is to consider a range of frequency bands (sufficiently narrow to allow for a correct estimation of the Hilbert envelope) and compute the envelope FC for each frequency band (Figure 3). This results in a series of frequency-specific Envelope FC matrices that can be used as a heuristic measure to fit resting-state models (Cabral et al., 2014b, Deco et al., 2016a) 
A

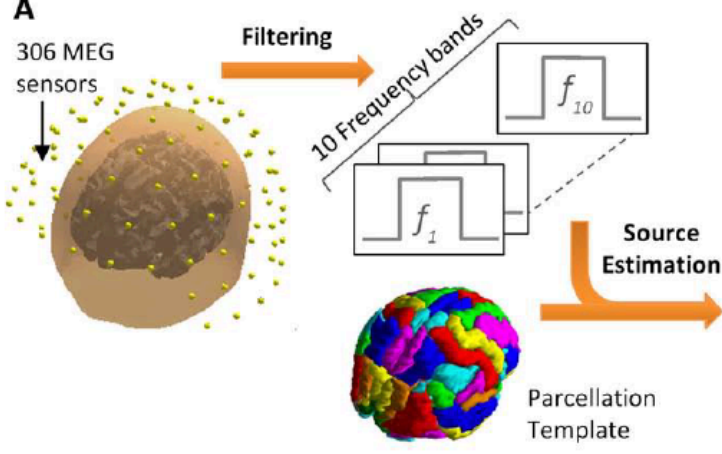

B

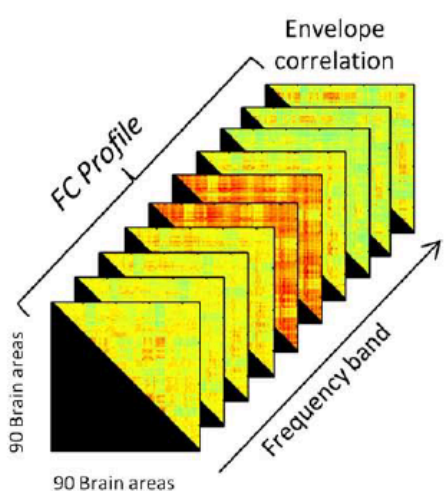

Figure 3 - Frequency-specific envelope FC from MEG data. (A) The MEG signals are filtered into different frequency bands and projected into a number of source locations using a beamformer technique. (B) For each frequency band, the amplitude envelopes are estimated for each brain area and the correlation matrix between envelopes is computed, resulting in a set of frequency-specific envelope FC matrices. Adapted from Cabral et al. (2014b).

\section{b. FC Dynamics}

In recent years, several works have shown that the Functional Connectome is not stationary, but instead evolves over time (Chang and Glover, 2010, Handwerker et al., 2012, Jones et al., 2012, Hutchison et al., 2013a, Hutchison et al., 2013b, Allen et al., 2014, Calhoun et al., 2014, Leonardi et al., 2014, Zalesky et al., 2014, Hansen et al., 2015). This has brought a new perspective to study resting-state activity by considering the temporal dimension of FC. In that direction, Calhoun et al. (2014) proposed the term Chronnectome to describe the time-varying properties of the Functional Connectome. Interestingly, the expanding idea is that the FC evolves as a multi-stable process passing through multiple and reoccurring discrete FC states, rather than varying in a more continuous sense (Hutchison et al., 2013a, Allen et al., 2014, Hansen et al., 2015, Preti et al., 2016). While different methodological approaches have been proposed to analyze the FC in the temporal domain (see Preti et al. (2016) for a comprehensive review) and to test for its statistical significance (Hutchison et al., 2013b, Hindriks et al., 2015a, Leonardi and Van De Ville, 2015, Zalesky and Breakspear, 2015), the best methodology to explore dynamic changes in FC is still to be decided. In the 
following, we briefly describe some of the methods to assess dynamic FC, which we find useful to apply in the analysis of whole-brain resting-state models.

\section{i. Assessing dynamic FC}

The most common and straightforward approach to investigate the temporal evolution of FC is the Sliding-Window Correlation (SWC) (Sakoglu et al., 2010, Handwerker et al., 2012, Jones et al., 2012, Hutchison et al., 2013b, Allen et al., 2014, Hansen et al., 2015). This is achieved by calculating the correlation matrix, $\mathrm{FC}(\mathrm{t})$, within a given time-window, shifting this window and recalculating the correlation matrix at time $t+1$ (see Figure 4 for an illustration). This procedure is successively repeated over the whole recording time, resulting in a time-varying $F C_{N x N x T}$ matrix (where $N$ is the number of brain areas and $T$ the number of time points). Yet, the fundamental nature of the SWC technique, implying the choice of a fixed window length, limits the analysis to the fluctuations in the frequency range below the window period, so the ideal window length to use remains under debate (Sakoglu et al., 2010)(Leonardi and Van De Ville, 2015, Zalesky and Breakspear, 2015, Laumann et al., 2016)(Hutchison et al., 2013a, Preti et al., 2016).

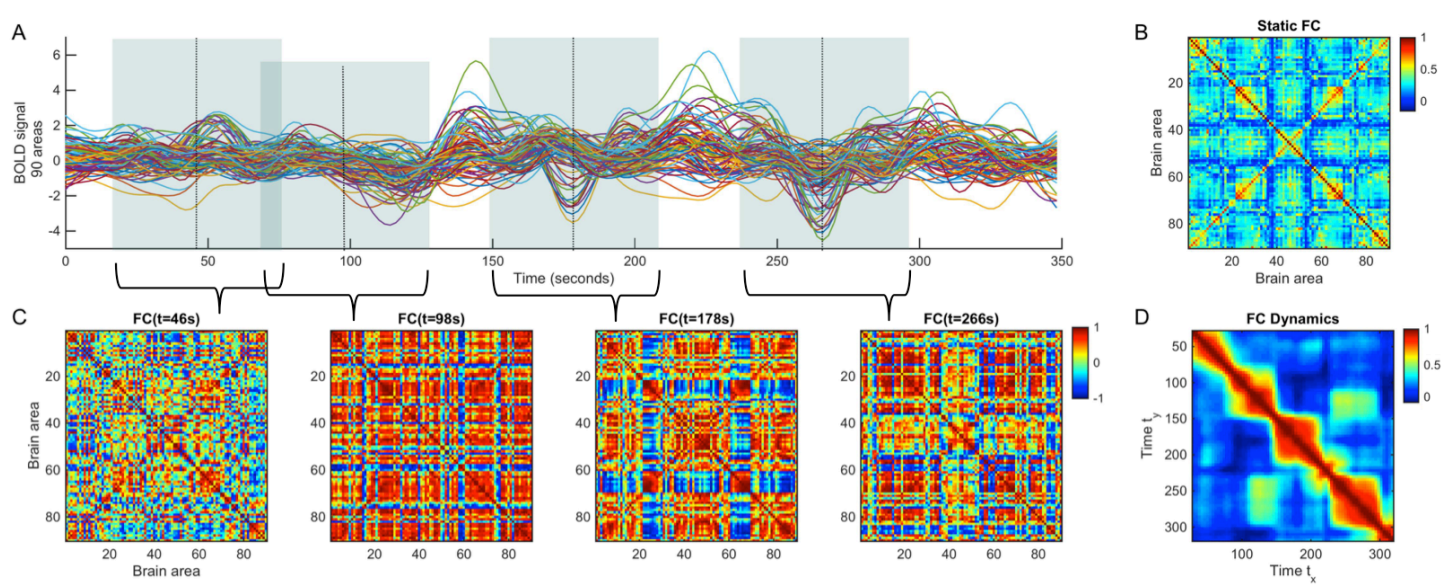

Figure 4 - FC dynamics based on sliding-window correlation. (A) BOLD signal at 90 locations from one healthy adult at rest (350seconds, TR=2s). (B) The static FC is computed as the correlation matrix $(90 \times 90)$ 
of BOLD time courses over the whole recording time. (C) Example of correlation matrices FC(t) obtained for sliding-windows of 60 seconds centered at different time points $t$. (D) The time-versus-time matrix of FC Dynamics, $F C D\left(t_{x}, t_{y}\right)$ is obtained by correlating any $F C\left(t_{x}\right)$ centered at time $t_{x}$ with any $F C\left(t_{y}\right)$ centered at time $t_{y}$.

Following the limitations of sliding-window analysis, a number of methods have been proposed to estimate the $\mathrm{FC}(\mathrm{t})$ at the instantaneous level. For instance, Coherence Connectivity (CC) consists in computing the phase coherence between time series at each recording frame (Glerean et al., 2012, Ponce-Alvarez et al., 2015, Deco and Kringelbach, 2016). In brief, the instantaneous BOLD phase of area $n$ at time $t, \theta_{n}(t)$, is estimated using the Hilbert transform, which expresses a given signal $x$ in polar coordinates as $x=A \cdot{ }^{*} \cos (\theta)$. Given the phase, the angle between two BOLD signals is given by their absolute phase difference: $\Theta_{n p}(t)=$ $\left|\theta_{n}(t)-\theta_{p}(t)\right|$. Then, the $C C(t)$ between a pair of brain areas $n$ and $p$ is calculated as:

$C C_{n p}(t)=\cos \left(\Theta_{n p}(t)\right), n, p \in N=1, \ldots, 90$

where values range between -1 (areas in anti-phase) and 1 (in-phase).

Another simple and quasi-instantaneous measure of coupling is the Multiplication of Temporal Derivatives (MTD) (Shine et al., 2015). The MTD measure consists in first calculating the temporal derivative $\left(T D_{n}\right)$ of each time series $\left(s_{n}\right)$ of length $t$ by performing a first-order differencing $T D_{n}=d s_{n} / d t$ (i.e. subtracting the value of $s(t-1)$ from the value of $s(t))$. For each pair of areas $n$ and $p$, the MTD at time $t$ is given by:

$M T D_{n p}(t)=\frac{T D_{n}(t) \times T D_{p}(t)}{S D_{n} \times S D_{p}}$

where $S D_{n}$ is the standard deviation of $T D_{n}$. Positive MTD scores reflect 'coupling' in the same direction of signal change across nodes, whereas negative scores 
reflect 'anti-coupling' (that is, signal in one node increasing while the other is decreasing).

Although instantaneous measures are more susceptible to high-frequency noise fluctuations, they can be smoothed (using e.g. a simple moving-average) without the methodological constrains imposed by the correlation measure.

Beyond the methods described above, other approaches worth noting include time-frequency analysis (Chang and Glover, 2010, Yaesoubi et al., 2015), phasedependent eigenconnectivities (Preti et al., 2016), or point process analysis (Liu and Duyn, 2013)(Tagliazucchi et al., 2012a).

\section{ii. FCD matrix}

To analyse the time-evolving FC matrices - defined either using SWC, CC, MTD or other - it is useful to compute a time-versus-time matrix representing the functional connectivity dynamics $(F C D)$, where each entry $F C D\left(t_{1}, t_{2}\right)$ is defined by a measure of resemblance between $\mathrm{FC}\left(\mathrm{t}_{1}\right)$ and $\mathrm{FC}\left(\mathrm{t}_{2}\right)$ (see Figure 4D). Since the $\mathrm{FC}(\mathrm{t})$ matrices are symmetric across the diagonal, resemblance is typically estimated between vectors containing all values above the diagonal (or below) of each FC( $\mathrm{t})$ matrix. This resemblance can be quantified using Pearson's correlation or cosine similarity (i.e. normalized inner product) between the $\mathrm{FC}(\mathrm{t})$ vectors at times $t_{1}$ and $t_{2}$. Epochs of stable $\mathrm{FC}$ patterns are reflected as square blocks around the FCD diagonal and reoccurrences of the same pattern appear as square blocks distant from the diagonal. Hence, the typical FCD matrix during the resting-state has a checkerboard appearance (Hutchison et al., 2013a, Hansen et al., 2015).

iii. FC states 
Although the FCD matrix contains rich information about the time-dependencies of the $\mathrm{FC}(\mathrm{t})$, in order to define a discrete number of FC states that reoccur over time and across subjects, it is necessary to the apply a clustering algorithm. The different FC states may then be characterized in different aspects, namely their spatial configuration, duration (or dwell time/lifetime), probability of occurrence (or fractional occupancy) or switching trajectories. Following the work of Allen et al. (2014), Hansen et al. (2015) applied unsupervised k-means clustering to all $\mathrm{FC}(\mathrm{t})$ across all subjects to characterize the switching dynamics of FC, which revealed a number of FC patterns that temporarily emerge -and often reoccur- within the same session. Using another approach, Baker et al. (2014) applied Hidden Markov Models (HMM) to the amplitude envelopes of MEG signals and identified fast transient FC states in spontaneous human brain activity with spatial topographies similar to those of well-known resting state networks. Notably, the FC states were found to last very shortly $(100-200 \mathrm{~ms})$, suggesting that the resting brain is constantly changing between different patterns of activity much more rapidly than previously thought (Vidaurre et al., 2016).

\section{Models of Resting-state activity}

Given the evident network dynamics emerging spontaneously at rest and its spatial similarity with the underlying structural connectivity, whole-brain network models are a useful tool to investigate the biophysical mechanisms underlying resting-state activity. By considering the dynamics emerging spontaneously from the interplay between brain areas when these are embedded in the neuroanatomical network, one can analyze the observed 
phenomena in the light of empirical data and formulate scenarios for the physiological origin of resting-state activity. This is achieved by considering a whole-brain network model where nodes refer to brain areas and links refer to the connections between them (Figure 5). The activity of each brain area is represented by one or more mathematical expressions representing the spontaneous behavior of isolated brain areas with an additive term representing the input received by anatomically connected areas (the main equations of the models discussed herein are reported in the Supplementary Information).

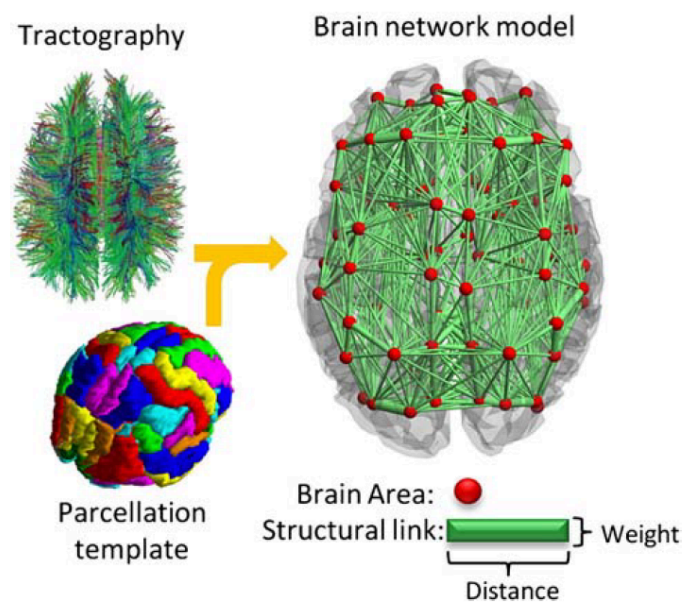

Figure 5 - In whole-brain network models, nodes refer to brain areas defined according to a given parcellation scheme, and links refer to the connections between them, typically obtained from DTI/DSI based tractography. Adapted from (Cabral et al., 2014b)

\section{a. Generative mechanistic scenarios}

Following different lines of thought, a variety of scenarios have been proposed for the genesis of resting-state activity, including multi-stability, supercritical bifurcations, chimera synchronization, self-organizing patterns, connectomespecific harmonics or simply correlated noise-induced deviations from the equilibrium (studies proposing these scenarios are reviewed in the following and summarized in Table 1). To test and validate these scenarios, theoreticians make use of computational models where similar behaviors can be obtained 
from very reduced network models. Depending on the reduction line, the node dynamics can be represented with different degree of biophysical realism and complexity, ranging from detailed populations of neurons with specific neurotransmitter receptor types, neural-field or neural-mass models, to phenomenological mesoscopic models. Importantly, different generative models may be used to test the same mechanistic scenario and vice-versa. As such, beyond their mathematical definitions (which can be found in detail in the Supplementary Information), we focus here on the mechanistic scenarios proposed by the different studies for the genesis of resting-state activity. In Table 1, we made an attempt to summarize and compare the different models focusing on their biophysical and dynamical properties. However, it is important to note that other relevant models (including variations of the ones considered herein) have been used to explain different phenomenological aspects of restingstate activity not considered in the current review (Petkoski and Jirsa, Freyer et al., 2011, Deco et al., 2012, Senden et al., 2012, Nakagawa et al., 2014, Hindriks et al., 2015b, Vasa et al., 2015, Vuksanovic and Hovel, 2015, Robinson et al., 2016). 


\begin{tabular}{|c|c|c|c|c|c|c|c|c|c|c|c|c|c|}
\hline & & 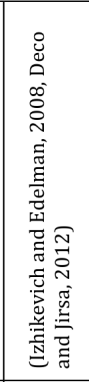 & 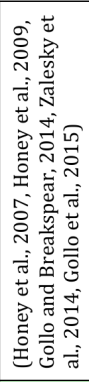 & 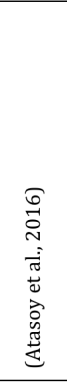 & 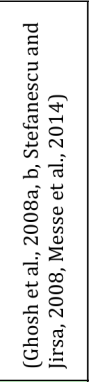 & 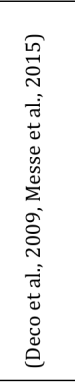 & 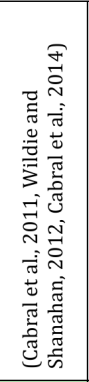 & 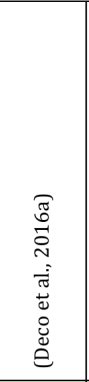 & 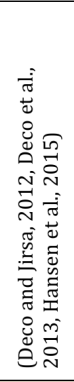 & 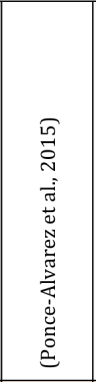 & 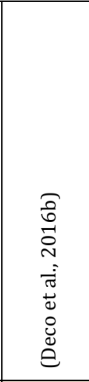 & 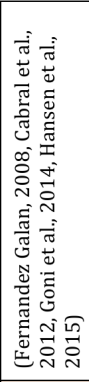 & 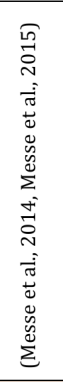 \\
\hline & 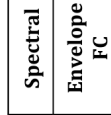 & $\sim$ & 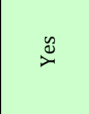 & .. & 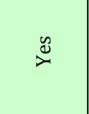 & .. & 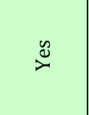 & 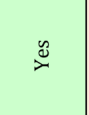 & z̊ & $\stackrel{2}{z}$ & \&̊ & $\stackrel{0}{z}$ & z̊ \\
\hline 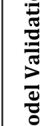 & 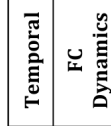 & $\stackrel{\check{\circlearrowright}}{\check{\nu}}$ & $\stackrel{\check{\varpi ̆}}{\rightleftharpoons}$ & . & $\therefore$ & 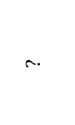 & $\stackrel{\mathscr{o ̆}}{\check{\nu}}$ & $\stackrel{\check{\nu}}{x}$ & 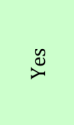 & $\stackrel{\mathscr{y}}{\check{x}}$ & $\stackrel{\mathscr{\nu}}{\check{\nu}}$ & 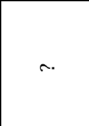 & $\stackrel{\circ}{z}$ \\
\hline & 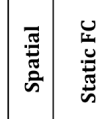 & $\stackrel{\circlearrowright}{\circlearrowright}$ & 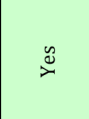 & $\stackrel{\check{\Xi}}{\check{\nu}}$ & 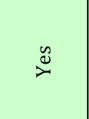 & $\stackrel{\check{\Delta}}{\searrow}$ & 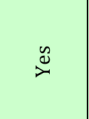 & 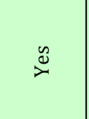 & $\stackrel{\mathscr{D}}{\check{\nu}}$ & 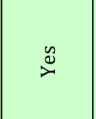 & $\stackrel{\mathscr{\nu}}{\not}$ & $\stackrel{\check{\nu}}{\check{\nu}}$ & $\stackrel{\mathscr{\vartheta}}{\check{0}}$ \\
\hline & 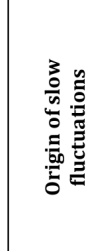 & 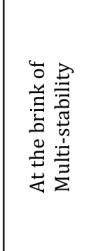 & 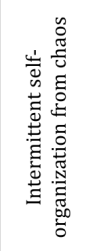 & 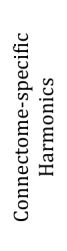 & 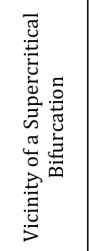 & 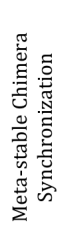 & 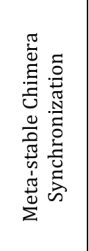 & 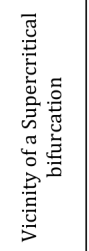 & 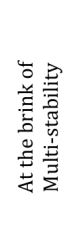 & 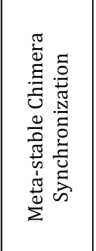 & 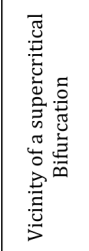 & 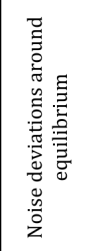 & \\
\hline 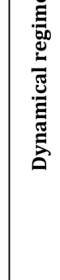 & 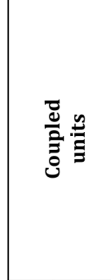 & 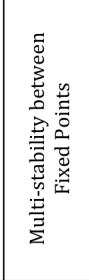 & 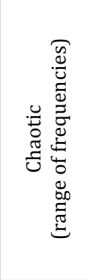 & 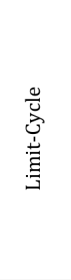 & 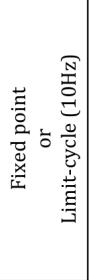 & 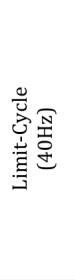 & 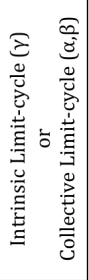 & 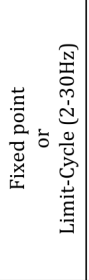 & 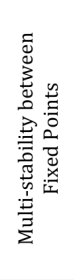 & 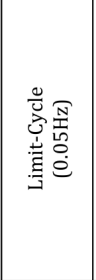 & 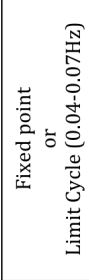 & 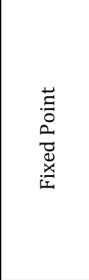 & \\
\hline & 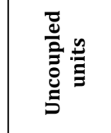 & $\begin{array}{l}\text { 䓂 } \\
\text { 咅 } \\
\text { 产 }\end{array}$ & 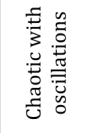 & 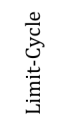 & 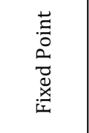 & 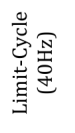 & 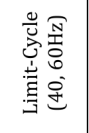 & 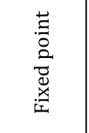 & $\begin{array}{l}\text { 䓂 } \\
\text { 言 } \\
\text { 产 } \\
\text { 产 }\end{array}$ & 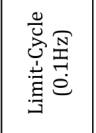 & 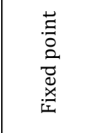 & 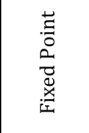 & \\
\hline & 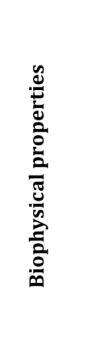 & 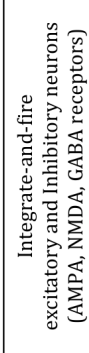 & 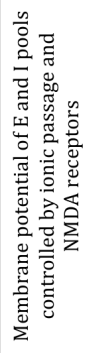 & 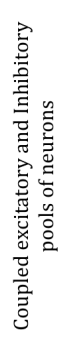 & 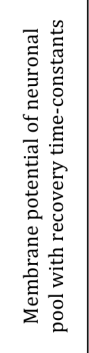 & 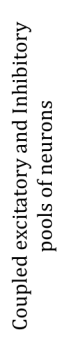 & 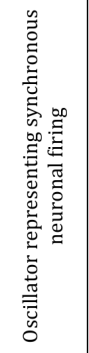 & 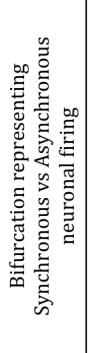 & 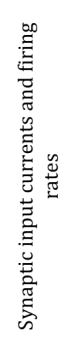 & 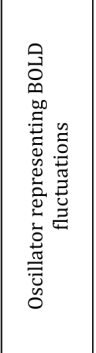 & 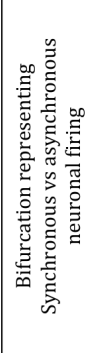 & 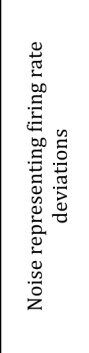 & 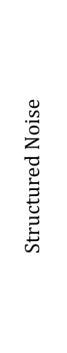 \\
\hline & & 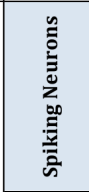 & 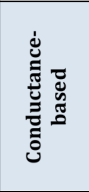 & 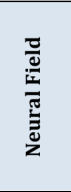 & 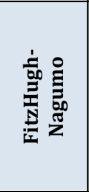 & 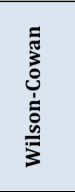 & 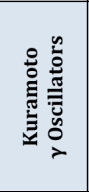 & 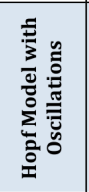 & 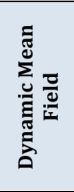 & 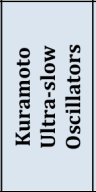 & 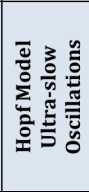 & 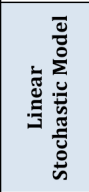 & 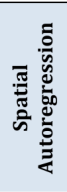 \\
\hline
\end{tabular}

Table 1 - Comparison between different models of resting-state in terms of biophysical properties, dynamical scenario and validation steps. 


\section{i. At the brink of multi-stability}

Starting with the most detailed generative model, Deco and Jirsa (2012) used a network of spiking neurons (Izhikevich and Edelman, 2008) representing each brain area as a pool of mutually interconnected excitatory and inhibitory neurons (Brunel and Wang, 2001). When uncoupled, the neural pools are characterized by a stable pattern of low firing activity in all cortical areas. As the coupling between areas increases, the collective behavior of spiking neurons can settle into new patterns of high firing activity (the so-called ghost attractors), while the state of low firing activity is still stable. In this critical range of coupling, the system is at the brink of multi-stability (where different stable states co-exist) and small noisy perturbations can induce transitions between states, leading to slow fluctuations that modulate the hemodynamic signal, reproducing the empirical FC obtained from fMRI.

With a similar bifurcation diagram, the Dynamic Mean Field Model (DMF) (Deco and Jirsa, 2012, Deco et al., 2013, Hansen et al., 2015) reduces the complexity of the spiking neurons model to a small set of differential equations representing the ensemble dynamics in each cortical area, leading to similar results.

In Hansen et al. (2015), the dynamic mean-field model was modified to adopt enhanced non-linearity in order to obtain a better fit with the FCD matrix.

In brief, these works propose a common scenario in which the brain is a multistable dynamical system and the phenomena observed at rest are the result of noise-driven explorations of its dynamical repertoire. So far, the dynamics occurring at the faster timescales of neurophysiological rhythms has not been explored. 


\section{ii. Intermittent self-organization from chaos}

Considering the biophysical properties of the neuronal membrane, the conductance-based model defines the membrane potential of excitatory and inhibitory populations of neurons as a function of ionic conductance through voltage-gated and ligand-gated ion channels as well as passive conductance of leaky ions (Hodgkin and Huxley, 1952, Breakspear et al., 2003, Honey et al., 2007, Honey et al., 2009). Simulated activity at the local level was described as chaotic with self-organizing patterns emerging spontaneously and intermittently from the chaotic system (Honey et al., 2007, Honey et al., 2009). Notably, when computing inter-area correlations between the simulated hemodynamic responses, significant agreement with FMRI FC was found.

Investigating the temporal dynamics of FC, Zalesky et al. (2014) used the conductance-based model on macaque connectivity and showed the emergence of coordinated fluctuations in FC akin to those seen in human data from the Human Connectome Project. Addressing the faster timescales of neurophysiological rhythms, Gollo and Breakspear (2014) and Gollo et al. (2014) found that, for intermediate coupling strengths where resonance and frustration coexist, different meta-stable synchronization patterns arise depending on the time delay between brain areas and the network size.

Taken together, these studies make proof of the powerful dynamical complexity of the conductance-based model to study the emergence of self-organizing patterns from chaos, a likely scenario to explain resting-state activity.

iii. In the vicinity of a supercritical bifurcation 
Adopting a perspective from dynamical systems' theory Ghosh et al. (2008b) proposed a reduced model where each brain area, when uncoupled, is a dynamical system operating in an equilibrium state (fixed point). On receiving sufficient input, the neural population is perturbed from its equilibrium state and starts oscillating (engaging in a limit-cycle). This type of behavior can be represented mathematically by a supercritical bifurcation, which represents the transition between a fixed point and a limit-cycle. The system returns to the equilibrium in a characteristic transient manner that depends on the dynamic repertoire of the system, which is shaped by the spatial and temporal properties of the SC (links and transmission delays). Given the frequency of oscillations observed with EEG (most power at $10 \mathrm{~Hz}$ during rest) and the large-scale structure of resting-state networks (up to $20 \mathrm{~cm}$ ), the authors report the importance of considering the time delays between brain areas induced by a finite propagation speed. The authors test this scenario with different neuralmass models with a supercritical bifurcation, namely Hopf oscillators, WilsonCowan systems, and FitzHugh-Nagumo systems, all providing similar results. In brief, in this approach each population is characterized by a degree of excitability, in which the increase of excitation parameterizes the onset of oscillations emerging from a quiescent state. When the populations are embedded in a network and operating near the critical boundary, oscillations emerge and dissipate, leading to slow envelope fluctuations shaped by the spacetime structure of the couplings.

This dynamical scenario was also considered in the works by Deco et al. (2016b) and (Deco et al., 2016a), where brain areas operate near the critical border of a supercritical Hopf bifurcation. While Ghosh et al. (2008b) consider a fundamental 
frequency at $10 \mathrm{~Hz}$ (to match the peak of EEG resting-state frequency spectra), Deco et al. (2016b) consider a fundamental frequency in the range of meaningful resting-state BOLD fluctuations, i.e. around $0.05 \mathrm{~Hz}$. Since it focused on the slow mesoscopic behavior observed in fMRI, time delays were neglected. Notably, simulations with this Ultra-slow Hopf model not only show an excellent fit with the empirical FC but also display a rich FC dynamics, with time-dependencies that fit the empirical observations.

More recently, Deco et al. (2016a) have proposed a Multi-frequency Hopf Model where this same scenario is contemplated but considering that brain areas can resonate at one or multiple fundamental frequencies, ranging between 2 and $30 \mathrm{~Hz}$ in agreement with the spectral profile of resting-state activity revealed by MEG. In this model, sporadic transitions to the oscillatory state are accompanied by an increase in the power of the corresponding oscillations, leading to slow and aperiodic envelope fluctuations of narrow-band carrier oscillations.

Exploring the network dynamics beyond the resting-state, Spiegler et al. (2016) investigated the result of focal stimulation on a whole-brain network model with long-range heterogeneous SC together with short-range homogeneous SC. At rest, the operating point of each network node is at the same distance to a supercritical Andronov-Hopf bifurcation. An excitatory stimulation pushes the system closer to criticality by selectively moving the operating point of particular network nodes closer to the Andronov-Hopf bifurcation. Because the stimulation propagates via the heterogeneous SC, some stimulation sites result in widespread and long-lasting patterns that shape dynamically responsive brain networks, some of which corresponding to known resting-state networks (Spiegler et al., 2016). 
In sum, this simple scenario where brain areas operate in the vicinity of a supercritical bifurcation provides a mechanistic explanation for the different spatial, temporal and spectral phenomena observed in the brain, not only at rest but also following stimulation, so it is likely to become a strong candidate in the new generation of whole-brain network models.

\section{iv. Meta-stable Chimera Synchronization}

From a different mechanistic perspective, Deco et al. (2009) considered the behavior of coupled Wilson-Cowan units with self-sustained oscillations in the gamma-frequency band in agreement with experimental (Buhl et al., 1998, Fisahn et al., 1998) and theoretical neurophysiological studies (Brunel and Wang, 2003). When coupled in a neuroanatomical network structure and for sufficiently weak coupling, different sub-networks can temporarily synchronize while the whole network never fully synchronizes (in a so-called Chimera regime)(Shanahan, 2010). With a sufficient degree of noise and for sufficiently large delays, the system switches between different partially synchronized network states (defined by the SC), which results in correlated BOLD signal within sub-networks.

In order to further explore the mechanisms of Chimera synchronization, Cabral et al. (2011) used coupled Kuramoto Gamma-band Oscillators to show that, even in the absence of noise, the space-time structure of the human brain network supports a robust meta-stable dynamics where different sub-networks temporally synchronize and desynchronize over time, on a time-scale much slower $(<0.1 \mathrm{~Hz})$ than the local fast gamma-band oscillations. This meta-stable regime (where partially synchronized states are only stable for a limited time 
period) induces spontaneous (noise-free) fluctuations in the synchrony degree of sub-networks leading to correlated slow BOLD-signal fluctuations (Cabral et al., 2011, Wildie and Shanahan, 2012).

In the light of MEG observations, metastable Chimera synchronization was further found to generate slow envelope fluctuations at different carrier frequencies fairly reproducing frequency-specific envelope FC (Cabral et al., 2014b). This occurs because brain areas alternate between their intrinsic limitcycle at $40 \mathrm{~Hz}$ and the collective limit-cycles of synchronized sub-networks (which occur at reduced frequencies due to the time delays between brain areas (Niebur et al., 1991)). In this scenario, resting-state brain rhythms are divided into: i) gamma-band oscillations as the intrinsic frequency of a brain area, ii) alpha- and beta-band oscillations as collective network frequencies, and iii) ultra-slow aperiodic envelope fluctuations generated by meta-stability of synchronized sub-systems.

More recently, Ponce-Alvarez et al. (2015) considered Ultra-slow Kuramoto Oscillators with fundamental frequency at $0.05 \mathrm{~Hz}$, revealing the transient formation and dissolution of multiple communities of synchronized brain regions. Although this approach explicitly neglected the contribution of faster neurophysiological rhythms, it found consistent approximation of the temporal and spatial FC patterns of empirical fMRI data.

In sum, these studies show that resting-state activity can be interpreted from the perspective of synchronization phenomena in complex networks.

\section{v. Connectome-specific harmonic waves}


Recently, Atasoy et al. (2016) demonstrated that the collective dynamics of human cortical activity at the macroscopic scale can be predicted by the eigendecomposition of the Laplace operator of the Structural Connectome. This mathematical framework lies at the heart of most self-organizing patterns in nature, including theories of heat, light, sound, electricity, magnetism, gravitation and fluid mechanics (Stewart, 1999). Remarkably, different resting-state networks are matched by the spatial patterns (Laplacian eigenfunctions) corresponding to certain natural frequencies (Laplacian eigenvalues) of the Structural Connectome (obtained at high-resolution, with more than 20,484 nodes)(Atasoy et al., 2016). This work presents evidence that Laplacian eigenfunctions can provide a simple yet almost universal description for patterns of synchrony throughout the cortex in the resting state. In order to demonstrate the emergence of these patterns from the cortico-cortical and thalamo-cortical interactions, the global network behaviour was modelled with a neural field model of coupled excitatory and inhibitory neural populations (see the Supplementary Information for further model details).

\section{vi. Noise deviations around the equilibrium}

A different approach to explain resting-state activity was proposed in Cabral et al. (2012) where noise-induced perturbations at rest are assumed to be so small that they only induce firing rate deviations around the stable asynchronous state (fixed point), with a characteristic exponentially decaying damping time-scale back to the fixed point. The firing rate deviations around the fixed point are described by a Linear Stochastic Model (see Supplementary Information), which can be reduced from a Wilson-Cowan system by removing the inhibitory 
populations and saturation function (Fernandez Galan, 2008, Goni et al., 2014). Since brain areas within a sub-network receive correlated noisy input from the structural connectivity, for a critical degree of coupling they display correlated fluctuations in firing rate, leading to a fair approximation of the empirical grandaverage FC (Cabral et al., 2012, Messe et al., 2014). However, the Linear Stochastic Model fails to reproduce the typical FCD patterns (Hansen et al., 2015) and faster neurophysiological rhythms are neglected.

\section{vii. Spatial autoregression}

Without any implicit dynamics, the simultaneous autoregressive (SAR) model expresses the BOLD signal as a purely linear combination of noise fluctuations in all regions (Messe et al., 2014, Messe et al., 2015). Notably, this 'model' performs as good or even better than other models in predicting the empirical FC from the SC (Messe et al., 2015). This happens because the ability of different models to predict the grand-average FC from SC can be reduced to a simple stationary linear process associated to the SC matrix that is implicit to all models. Although this study is important to depict the core similarity between all models, the investigation of the genesis of resting-state activity cannot be assessed in phenomenological terms with this model, since it disregards any dynamical aspect.

\section{b. Validation of Resting-state models}

With the availability of human Structural Connectivity and the corresponding fMRI-based Functional Connectivity, a number of whole-brain computational models have focused in predicting the FC from the underlying $\mathrm{SC}$ and thus, led to the idea that model performance consists in optimizing the correlation between 
the simulated and empirical FC patterns (Honey et al., 2009, Cabral et al., 2011, Deco et al., 2013, Messe et al., 2015, Ponce-Alvarez et al., 2015). This has misled some to believe that models serve (only) as a tool to predict function from structure (Messe et al., 2014), disregarding the powerful capacity of bottom-up computational models to investigate physiological mechanisms occurring in nature. Indeed, Messe et al. (2015) have shown that even a linear stochastic model can successfully predict the grand-average FC. Although this simple model can be useful to make specific predictions of SC effects on FC, it does not generate any tyoe of dynamics so it is useless in phenomenological terms.

Before the availability of whole-brain human SC-FC, the models were adjusted to fit other aspects of resting-state dynamics, namely in terms of complexity, number of components, cluster synchronization and frequency of slow fluctuations (Honey et al., 2007, Ghosh et al., 2008a, b, Deco et al., 2009). The focus of such whole-brain network models was to investigate the biophysical mechanisms leading to the emergence of slow spatiotemporal patterns, without any heuristic measure to fit beyond the $0.1 \mathrm{~Hz}$. Notably, all these models were later found to successfully predict the FC from SC, reinforcing their phenomenological potential (Messe et al., 2015).

Given the growing experimental evidence reviewed in the previous sections and the wide range of candidate scenarios described above, we consider that restingstate models must go through a common set of validation steps in order to refresh the list of accepted candidates. We consider this validation must contemplate 3 dimensions: Spatial, Spectral and Temporal (in Table $\mathbf{1}$ we report whether any of these 3 validation steps has been successfully addressed):

\section{i. Spatial Validation}


The Grand-average FC matrix contains important spatial information regarding which areas appear more correlated over time. This matrix is strongly linked with the SC and the accuracy of the fitting between simulated and empirical FC patterns may vary widely according to the parcellation considered or to the quality of the SC, so any quantitative comparison between models must rely on the same empirical data. In other words, in purely bottom-up models, the lack of important inter-hemispheric links in the SC will impose a limit up to which models can reach in fitting the FC. The same occurs if important networks structures are not captured with sufficient detail in the parcellation scheme. Nevertheless, if caution is taken in its interpretation, the prediction of FC patterns in spatial terms should remain an important step towards the heuristic validation of computational models.

Beyond the FC matrix, the spatial maps of resting-state networks have been consistently identified in fMRI studies using ICA-based methods (Beckmann et al., 2005, Damoiseaux et al., 2006). Moreover, the same resting-state networks have been identified by applying ICA to the power envelopes of MEG signals (Brookes et al., 2011b). As such, an additional step towards the validation of resting-state models in spatial terms would be the detection of meaningful functional networks using ICA.

\section{ii. Temporal Validation}

As discussed above, resting-state FC is known to evolve over time, with timedependencies being revealed by reoccurring patterns in the FCD matrix. However, the methods for the analysis of FCD have only recently begun to get established. While there is controversy regarding the accurate methods to 
measure time-evolving FC (either using sliding-window correlation, coherence connectivity or multiplication of temporal derivatives), it is important to verify which of the existing models are able to replicate the checkerboard pattern of FCD matrices. Since the FCD matrices are computed on a time-versus-time basis, comparison between simulated and empirical FCD matrices cannot be performed using similarity or correlation measures. As such, the current approach is to compare the histograms of FCD values (Hansen et al., 2015, Deco et al., 2016a, Deco and Kringelbach, 2016, Deco et al., 2016b). Indeed, the difference between FCD histograms has shown to be a sensitive measure to fit the models as it considers the time-dependencies of the FC (Figure 6). If the patterns of $\mathrm{FC}(\mathrm{t})$ are constant over time (i.e. no $\mathrm{FC}$ dynamics), the histogram of FCD values appears shifted to 1 . On the other hand, if $\mathrm{FC}(\mathrm{t})$ patterns are unrelated over time, the FCD distributions are shifted to zero. Instead, the histogram of empirical resting-state FCD matrices (shown in Figure 6B) displays a peak at intermediate values (corresponding to the periods of weak temporal similarities) together with a long tail towards high FCD values, indicating the existence of periods of high similarity between $\mathrm{FC}(\mathrm{t})$ patterns. Computing the Kolmogorov-Smirnov distance (KS-distance) between empirical and simulated FCD histograms (Figure 6A, blue line) allows searching for the range of parameters where the models generate time-evolving $\mathrm{FC}(\mathrm{t})$ patterns with temporal similarities matching the empirical ones (Hansen et al., 2015, Deco et al., 2016a, Deco and Kringelbach, 2016, Deco et al., 2016b). 
A

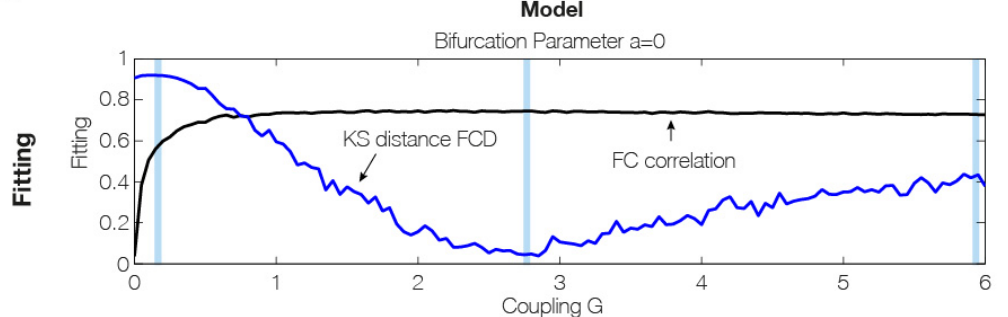

$\Delta$
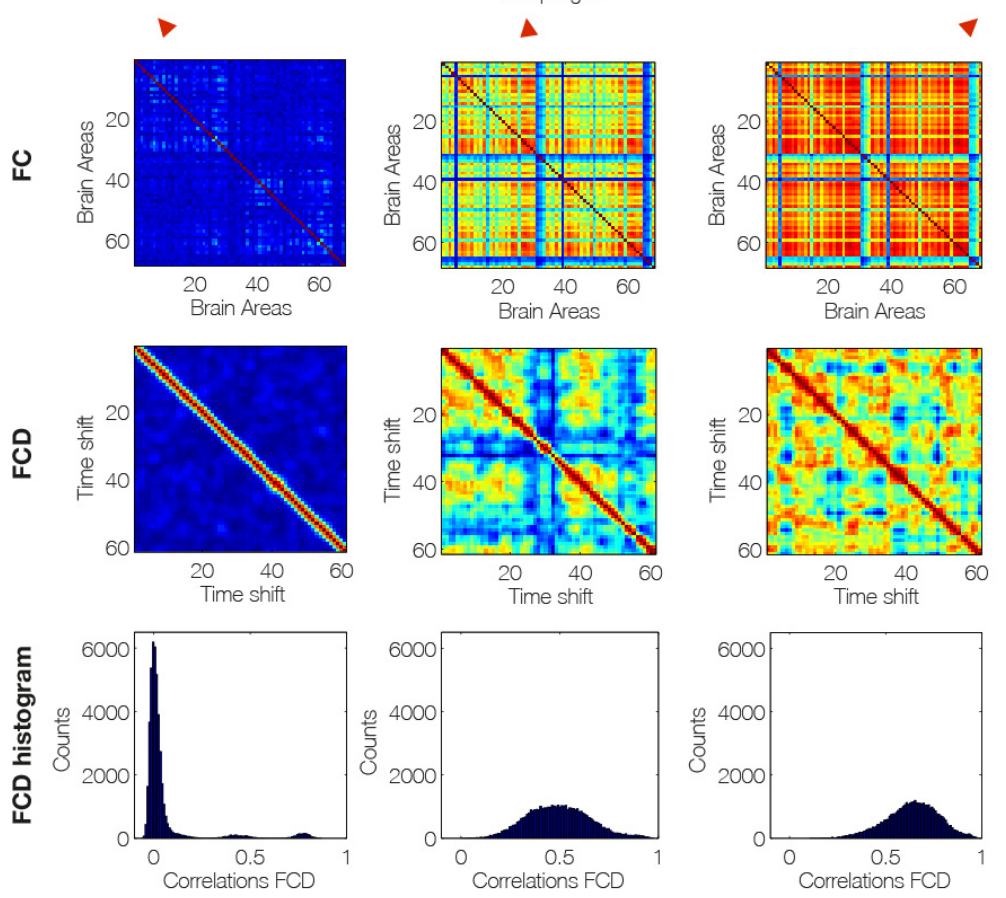

B

Data
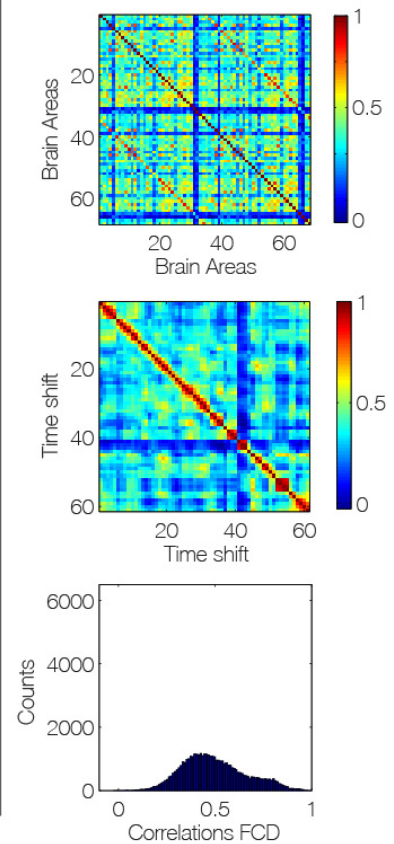

Figure 6 - Fitting the model to the histogram of FCD values. (A) The figure shows the fitting of the Hopf Model (Deco et al., 2016b) to empirical fMRI data as a function of the global coupling parameter, G. The fitting is evaluated either as the correlation with the empirical FC matrix (black line) or as the KS-distance between histograms of FCD values (blue line). Below, the grand-average FC matrix, FCD matrix and FCD histogram are shown for 3 working points (weak, optimal and strong G). (B) Empirical FC and FCD matrices together with the FCD histogram obtained from a single healthy subject. Figure reproduced from (Deco et al., 2016b).

Although the histogram of FCD values provides valuable information regarding the temporal dependencies of FC patterns in general terms, it misses several aspects of the FC dynamics, namely the characterization of the different FC patterns in terms of lifetime, probability and switching profiles. In that sense, assessing different FC states by applying a k-means clustering or HMM to simulated data would provide an important validation of the FC dynamics generated by resting-state models (Allen et al., 2014, Baker et al., 2014, Hansen et al., 2015). 


\section{iii. Spectral Validation}

We understand by spectral validation any measure that considers the frequency spectrum of resting-state rhythms, ranging from the fast oscillations observed with electrophysiology, EEG and MEG (mainly in the alpha, beta and gamma ranges) to the slow aperiodic fluctuations of BOLD signals and narrow-band envelopes (typically below $0.1 \mathrm{~Hz}$ ) (Engel et al., 2013). Although most models have proposed mechanisms for the origin of slow aperiodic BOLD signal fluctuations, the dynamics at the local level has been left largely unconstrained. Messe et al. (2015) have made an attempt to compare the power spectra of simulated data obtained with different computational models showing a variety of oscillatory behaviors (Figure 7Error! Reference source not found.). While the aim of resting-state models is not necessarily to quantitatively replicate the power spectrum of brain activity during rest, it is important to consider it at least qualitatively, in order to investigate the link between slow envelope fluctuations and the underlying oscillatory activity observed in resting-state MEG studies.

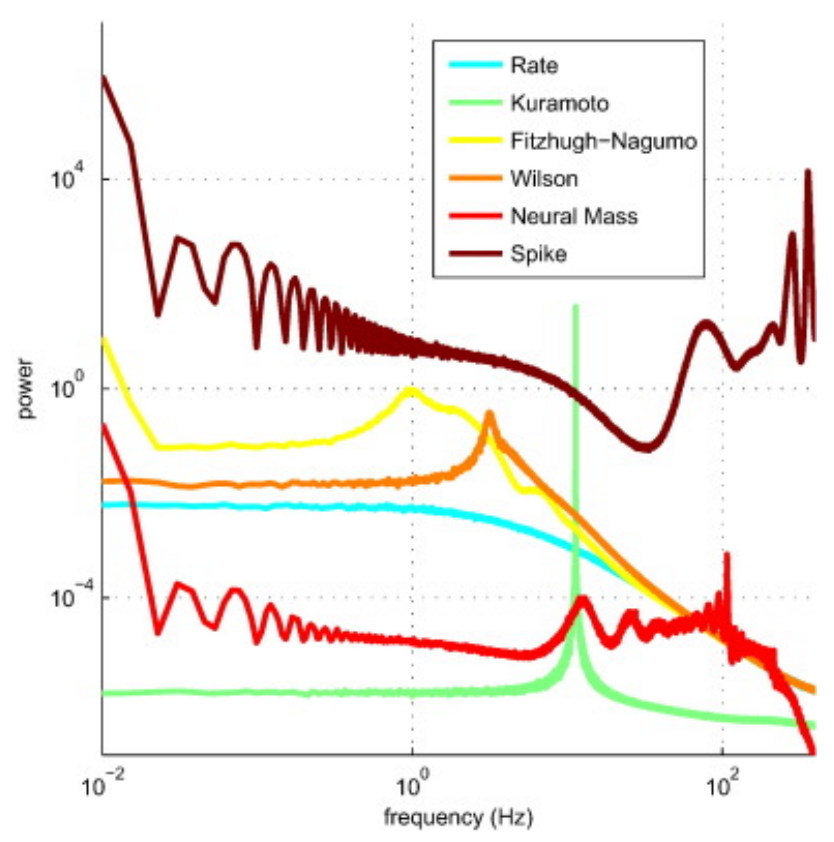


Figure 7 - Power spectra of simulated activity obtained with different computational models (Messe et al., 2015). Spectra were obtained at the point of optimal fit with the grand-average FC matrix and averaged across all regions. Figure reproduced from (Messe et al., 2015).

One approach to perform a validation in the spectral domain is to obtain the frequency-specific envelope FC matrices (see section 2.a) and compare with the ones obtained empirically from MEG (Cabral et al., 2014b, Deco et al., 2016a). Importantly, rather than aiming at an optimal quantitative fit (which largely depends on the bands and the number of brain areas considered), this measure should be used to search for the range of parameters where the models better explain the data in phenomenological terms.

\section{c. A new generation of resting-state models}

By refreshing the list of candidate models of resting-state activity through the incorporation of the spatial, temporal and spectral constrains proposed herein, a better insight about the mechanisms occurring in the complexity of the resting brain may be assessed. As can be seen in Table 1, different models have shown to generate simulated brain activity with a) a grand-average FC matrix of BOLD signals fitting the empirical one, b) slow aperiodic power fluctuations of faster oscillatory signals, and/or c) time-evolving patterns of FC. Since all models achieve a fairly good prediction of the static FC, the selective process of candidate models occurs at the level of FC Dynamics and Envelope FC.

In general terms, FC Dynamics is obtained when the simulated dynamics displays different (at least two) states of FC and the system switches between FC states on a slow time-scale. This is achieved when models operate in a critical regime, such that sporadic transitions at the critical border induce a reconfiguration of FC patterns. Notably, most of the proposed mechanistic scenarios operate at the edge of criticality, so apart from the Spatial Autoregressive Model (Messe et al., 
2014) which has clearly no implicit dynamics, all the other models -even the ones not specifically tested for Dynamic FC- are likely to display timedependencies in the FCD matrix. Note however, that when the model parameters have been optimized to fit the static FC (which occurs for a much broader range of parameters), it is likely that they need to be further adjusted in order to match the histogram of empirical FCD values (see Figure 6). For instance, in Hansen et al. (2015), the parameters of the dynamical mean-field model were slightly modified to enhance non-linearities and obtain a better fit of the empirical FC Dynamics.

Regarding the spectral validation, Envelope FC can only be meaningfully obtained if a) the models generate oscillations in the range of neurophysiological rhythms and b) the power of these oscillations is modulated over space and time by the SC. Even if arising from different generative mechanisms, several models display oscillations at the node level, namely the conductance-based model (Honey et al., 2007), the FitzHugh-Nagumo model (Ghosh et al., 2008b), the Hopf model (Deco et al., 2016a), the Wilson-Cowan model (Deco et al., 2009) and the Kuramoto oscillators (Cabral et al., 2014b). Fluctuations in the power of such oscillations occur due to the criticality of the system and can be divided into 2 main scenarios: a) damped oscillations -i.e. the oscillations emerge and dissipate from a quiescent equilibrium state - (Honey et al., 2007, Ghosh et al., 2008b, Deco et al., 2016a) or b) frequency modulation of self-sustained oscillations due to metastable synchronization in the presence of time-delays (Cabral et al., 2014b).

In addition, while in more mesoscopic models the fast oscillations were explicitly neglected (Cabral et al., 2012, Ponce-Alvarez et al., 2015, Deco et al., 2016b), they 
have been simply disregarded in the network of spiking neurons (Deco and Jirsa, 2012) or not specifically addressed in the neural field model by Atasoy et al. (2016), so these models may be adapted and/or extended to include oscillations and eventually display realistic Envelope FC patterns.

In conclusion, while we are not able in the current review to predict which mechanism is more likely to be at the genesis of resting-state activity, we can predict which ones will most likely fail and hence restrict the list of candidate scenarios. Indeed, models that explicitly neglected the faster time-scales of neurophysiological rhythms focusing solely on fMRI observations, namely the Spatial Autoregressive Model, the Linear Stochastic Model, and the Hopf or Kuramoto Models with only Ultra-Slow $(<0.1 \mathrm{~Hz})$ oscillations are likely to be left out, since they do not allow addressing all the time-scales implicated in restingstate activity, following to the latest empirical observations.

\section{Discussion}

Resting-state activity has been the subject of investigation of many experimental and theoretical works not only for its intriguing network dynamics but also for its implications in the brain's functional organization. Beyond the static correlation between BOLD signals, deeper insights into the FC dynamics at rest and the investigation of faster oscillatory components using MEG have unveiled new features of resting-state dynamics that can serve as novel constrains to depict the genesis of resting-state activity and, ultimately, better understand the dynamical mechanisms underlying functional connectivity in the brain.

\section{A single SC for multiple FCs}


From the perspective of dynamical systems' theory, the live brain can be seen as a system exhibiting multi-stability: the same neuroanatomical structure supports different stable dynamical states, each characterized by the activation of a specific functionally-relevant network (i.e. visual network, motor network, attention network, default mode network, etc.). Sustained by neuroimaging evidence it is now widely accepted that the brain uses a combination of specialized local processing (segregation) and large-scale functional assimilation (integration) to transform brain signals into thoughts or actions (Sporns, 2011). On this basis, segregated brain areas make use of the white matter anatomical pathways in order to form temporary functional coalitions to convey information. However, how brain areas selectively couple to each other to form different functional networks remains unclear.

\section{The importance of the resting-state}

Even if no specific task is being undertaken while we are at rest, the brain activity in this specific state has unveiled intriguing dynamical features leading to a paradigm shift in the way we look at brain function. Indeed, instead of being in a stable low firing equilibrium while waiting for a new trigger, the brain displays the activation of different functionally-meaningful networks of brain areas, which are believed to reflect the dynamical repertoire of macro-scale brain states, intrinsically shaped by the neuroanatomical structure (Tognoli and Kelso, 2014, Deco and Kringelbach, 2016).

Following the growing number of studies of resting-state activity, a number of theoretical works have aimed at investigating the biophysical mechanisms leading to the genesis of a similar dynamics in reduced network models with 
realistic (but low-dimensional) cortico-cortical connectivity. Since complex network dynamics cannot be easily assessed analytically, theoretical works resource to computational models in order to simulate the interplay between coupled neural masses. Importantly, modeling the resting-state should be seen only as a starting point (yet crucial) to understand brain network dynamics. Indeed, the resting brain can rapidly switch to a task by activating a functionallyrelevant network in response to a specific trigger. As such, modeling the brain as a dynamical system must ultimately consider its dynamical flexibility to rapidly activate a specific brain state in response to a specific input. However, in order to achieve such goal, it is important to first obtain an accurate picture of the complex network dynamics occurring in the brain at rest.

\section{Validation of resting-state models}

With the recent but imperative inclusion of the temporal dimension in the analysis of resting-state FC and with the insights from MEG studies, existing dynamical models of spontaneous activity need to be re-evaluated. Here, we provide an overview of the different steps to analyse the FCD or envelope FC, so that models can be revised in order to consider the novel constrains imposed by the latest empirical evidences. Indeed, even if these constrains were not imposed when the models were developed, and even if some adaptations must be undertaken, their mechanistic scenarios may remain valid in phenomenological terms. For instance, the networks of spiking neurons used by Deco and Jirsa (2012) may display oscillatory behaviour in some of the ghost attractor states, and therefore reveal power fluctuations in the dynamics that were not considered in the analysis. Similarly, the self-organizing patterns 
observed with the conductance-based model by Honey et al. (2009) may also reveal power fluctuations in a given frequency band. In terms of FC dynamics, the Kuramoto model with gamma oscillators (Cabral et al., 2014b) is likely to display different FC patterns when the different sub-networks synchronize. Therefore, all these models may potentially survive the validation process. Moreover, even a reduced model with no implicit neurophysiological rhythms may be adapted to include oscillations, without directly discarding its phenomenological scenario. Nevertheless, we can expect that a purely linear model without any implicit dynamics such as the SAR (Messe et al., 2014) is likely to fail in this validation process.

\section{Mechanisms of resting-state activity}

In this work we attempted to characterize the different models of resting-state activity according to the dynamical scenario they propose for the genesis of resting-state activity. From a general perspective, uncoupled nodes can be in 3 different states:

- Fixed-point: where the neurons fire in an asynchronous fashion represented as white noise.

- Limit-cycle: where neurons within a brain area are engaged in selfsustained oscillations

- Chaotic: where the diversity of biophysical parameters (i.e. membrane conductance, time constants, etc.) generates a complex dynamics.

On receiving input through the neuroanatomical network, this input may be sufficiently strong to induce transitions (from fixed point to limit cycle in the case of a supercritical bifurcation, or between different fixed points in the case of 
multiple fixed-point attractors), or sufficiently weak so that it only induces deviations from the fixed point. In the case of partial synchronization, the input from connected areas should be only sufficiently strong to induce the synchronization of sub-networks, but sufficiently weak to avoid the full synchronization of the whole-brain. Still in the case of synchronization, the switch between different synchronized subsystems may be driven by noise (Deco et al., 2009) or intrinsically induced (metastability) by heterogeneity in the system (Cabral et al., 2011, Cabral et al., 2014b, Ponce-Alvarez et al., 2015). Regarding the origin of slowness in the system leading to slow BOLD or envelope fluctuations, these originate from different mechanisms including stochastic effects (i.e. noise is tuned such that transitions only occur sporadically), to the distance between attractors (the further they are the longer it takes for a transition to occur spontaneously), due to the damping time-scale of the system (in the case of the rate model), or due to sufficiently weak coupling in a network with heterogenous delays/frequencies (such that synchronized subsystems are only stable for a limited period, or meta-state).

\section{Future perspectives}

Even with a refreshed list of new-generation resting-state models, it is likely that different mechanistic scenarios will remain acceptable. Beyond further validation procedures in the resting-state, the next step appears to be to start perturbing the models in order to investigate their response to stimulation, namely with the activation/stabilization of meaningful functional networks (Cocchi et al., 2015, Gollo et al., 2016, Spiegler et al., 2016). It is likely that such approach will bring a paradigm shift in whole-brain network models, which, 
rather than being termed Resting-State Models, will become a new generation of Whole-Brain Functional Models. Such models have the potential of becoming increasingly helpful in the preparation of clinical interventions, including brain stimulation techniques such as transcranial magnetic/electrical stimulation or deep brain stimulation.

\section{Acknowledgements}

JC and MLK were supported by the ERC Consolidator Grant CAREGIVING (n. 615539) and the TrygFonden Charitable Foundation. GD was supported by the ERC Advanced Grant: DYSTRUCTURE (n. 295129) and by the Spanish Research Project PSI2013-42091-P.

\section{References}

Aertsen AM, Gerstein GL, Habib MK, Palm G (1989) Dynamics of neuronal firing correlation: modulation of "effective connectivity". Journal of neurophysiology 61:900-917.

Allen EA, Damaraju E, Plis SM, Erhardt EB, Eichele T, Calhoun VD (2014) Tracking whole-brain connectivity dynamics in the resting state. Cerebral cortex 24:663-676.

Atasoy S, Donnelly I, Pearson J (2016) Human brain networks function in connectome-specific harmonic waves. Nature communications 7:10340.

Baker AP, Brookes MJ, Rezek IA, Smith SM, Behrens T, Probert Smith PJ, Woolrich M (2014) Fast transient networks in spontaneous human brain activity. eLife 3:e01867.

Bartzokis G, Cummings JL, Sultzer D, Henderson VW, Nuechterlein KH, Mintz J (2003) White matter structural integrity in healthy aging adults and patients with Alzheimer disease: a magnetic resonance imaging study. Archives of neurology 60:393-398.

Beckmann CF, DeLuca M, Devlin JT, Smith SM (2005) Investigations into restingstate connectivity using independent component analysis. Philosophical transactions of the Royal Society of London Series B, Biological sciences 360:1001-1013.

Biswal B, Yetkin FZ, Haughton VM, Hyde JS (1995) Functional connectivity in the motor cortex of resting human brain using echo-planar MRI. Magnetic resonance in medicine 34:537-541.

Biswal BB, Mennes M, Zuo XN, Gohel S, Kelly C, Smith SM, Beckmann CF, Adelstein JS, Buckner RL, Colcombe S, Dogonowski AM, Ernst M, Fair D, Hampson M, Hoptman MJ, Hyde JS, Kiviniemi VJ, Kotter R, Li SJ, Lin CP, 
Lowe MJ, Mackay C, Madden DJ, Madsen KH, Margulies DS, Mayberg HS, McMahon K, Monk CS, Mostofsky SH, Nagel BJ, Pekar JJ, Peltier SJ, Petersen SE, Riedl V, Rombouts SA, Rypma B, Schlaggar BL, Schmidt S, Seidler RD, Siegle GJ, Sorg C, Teng GJ, Veijola J, Villringer A, Walter M, Wang L, Weng XC, Whitfield-Gabrieli S, Williamson P, Windischberger C, Zang YF, Zhang HY, Castellanos FX, Milham MP (2010) Toward discovery science of human brain function. Proceedings of the National Academy of Sciences of the United States of America 107:4734-4739.

Breakspear M, Terry JR, Friston KJ (2003) Modulation of excitatory synaptic coupling facilitates synchronization and complex dynamics in a biophysical model of neuronal dynamics. Network 14:703-732.

Brookes MJ, Hale JR, Zumer JM, Stevenson CM, Francis ST, Barnes GR, Owen JP, Morris PG, Nagarajan SS (2011a) Measuring functional connectivity using MEG: methodology and comparison with fcMRI. NeuroImage 56:1082-1104.

Brookes MJ, Woolrich M, Luckhoo H, Price D, Hale JR, Stephenson MC, Barnes GR, Smith SM, Morris PG (2011b) Investigating the electrophysiological basis of resting state networks using magnetoencephalography. Proceedings of the National Academy of Sciences of the United States of America 108:16783-16788.

Brunel N, Wang XJ (2001) Effects of neuromodulation in a cortical network model of object working memory dominated by recurrent inhibition. Journal of computational neuroscience 11:63-85.

Brunel N, Wang XJ (2003) What determines the frequency of fast network oscillations with irregular neural discharges? I. Synaptic dynamics and excitation-inhibition balance. Journal of neurophysiology 90:415-430.

Buhl EH, Tamas G, Fisahn A (1998) Cholinergic activation and tonic excitation induce persistent gamma oscillations in mouse somatosensory cortex in vitro. The Journal of physiology 513 ( Pt 1):117-126.

Cabral J, Hugues E, Kringelbach ML, Deco G (2012) Modeling the outcome of structural disconnection on resting-state functional connectivity. NeuroImage 62:1342-1353.

Cabral J, Hugues E, Sporns O, Deco G (2011) Role of local network oscillations in resting-state functional connectivity. NeuroImage 57:130-139.

Cabral J, Kringelbach ML, Deco G (2014a) Exploring the network dynamics underlying brain activity during rest. Progress in neurobiology 114:102-131.

Cabral J, Luckhoo H, Woolrich M, Joensson M, Mohseni H, Baker A, Kringelbach ML, Deco G (2014b) Exploring mechanisms of spontaneous functional connectivity in MEG: how delayed network interactions lead to structured amplitude envelopes of band-pass filtered oscillations. NeuroImage 90:423435.

Calhoun VD, Miller R, Pearlson G, Adali T (2014) The chronnectome: time-varying connectivity networks as the next frontier in fMRI data discovery. Neuron 84:262-274.

Chang C, Glover GH (2010) Time-frequency dynamics of resting-state brain connectivity measured with fMRI. NeuroImage 50:81-98.

Cocchi L, Sale MV, Lord A, Zalesky A, Breakspear M, Mattingley JB (2015) Dissociable effects of local inhibitory and excitatory theta-burst stimulation on large-scale brain dynamics. Journal of neurophysiology 113:3375-3385.

Damoiseaux JS, Rombouts SA, Barkhof F, Scheltens P, Stam CJ, Smith SM, Beckmann CF (2006) Consistent resting-state networks across healthy 
subjects. Proceedings of the National Academy of Sciences of the United States of America 103:13848-13853.

de Pasquale F, Della Penna S, Snyder AZ, Lewis C, Mantini D, Marzetti L, Belardinelli P, Ciancetta L, Pizzella V, Romani GL, Corbetta M (2010) Temporal dynamics of spontaneous MEG activity in brain networks. Proceedings of the National Academy of Sciences of the United States of America 107:6040-6045.

Deco G, Cabral J, Woolrich M, Stevner A, Van Hartevelt TJ, Kringelbach ML (2016a) Single or Multi-Frequency Generators in On-going Brain Activity: a Mechanistic Whole-Brain Model following empirical MEG evidences. bioRxiv 084103.

Deco G, Jirsa V, McIntosh AR, Sporns O, Kotter R (2009) Key role of coupling, delay, and noise in resting brain fluctuations. Proceedings of the National Academy of Sciences of the United States of America 106:10302-10307.

Deco G, Jirsa VK (2012) Ongoing cortical activity at rest: criticality, multistability, and ghost attractors. The Journal of neuroscience : the official journal of the Society for Neuroscience 32:3366-3375.

Deco G, Jirsa VK, McIntosh AR (2011) Emerging concepts for the dynamical organization of resting-state activity in the brain. Nature reviews Neuroscience 12:43-56.

Deco G, Kringelbach M (2016) Metastability and Coherence: Extending the Communication through Coherence Hypothesis Using a Whole-Brain Computational Perspective. Trends in neurosciences 39:432.

Deco G, Kringelbach ML, Jirsa V, Ritter P (2016b) The dynamics of resting fluctuations in the brain: metastability and its dynamical cortical core. Biorxiv.

Deco G, Ponce-Alvarez A, Mantini D, Romani GL, Hagmann P, Corbetta M (2013) Resting-state functional connectivity emerges from structurally and dynamically shaped slow linear fluctuations. The Journal of neuroscience : the official journal of the Society for Neuroscience 33:11239-11252.

Deco G, Senden M, Jirsa V (2012) How anatomy shapes dynamics: a semi-analytical study of the brain at rest by a simple spin model. Frontiers in computational neuroscience 6:68.

Engel AK, Gerloff C, Hilgetag CC, Nolte G (2013) Intrinsic coupling modes: multiscale interactions in ongoing brain activity. Neuron 80:867-886.

Fernandez Galan R (2008) On how network architecture determines the dominant patterns of spontaneous neural activity. PloS one $3: \mathrm{e} 2148$.

Fisahn A, Pike FG, Buhl EH, Paulsen O (1998) Cholinergic induction of network oscillations at $40 \mathrm{~Hz}$ in the hippocampus in vitro. Nature 394:186-189.

Freyer F, Roberts JA, Becker R, Robinson PA, Ritter P, Breakspear M (2011) Biophysical mechanisms of multistability in resting-state cortical rhythms. The Journal of neuroscience : the official journal of the Society for Neuroscience 31:6353-6361.

Friston KJ, Frith CD, Liddle PF, Frackowiak RS (1993) Functional connectivity: the principal-component analysis of large (PET) data sets. Journal of cerebral blood flow and metabolism : official journal of the International Society of Cerebral Blood Flow and Metabolism 13:5-14.

Ghosh A, Rho Y, McIntosh AR, Kotter R, Jirsa VK (2008a) Cortical network dynamics with time delays reveals functional connectivity in the resting brain. Cognitive neurodynamics 2:115-120. 
Ghosh A, Rho Y, McIntosh AR, Kotter R, Jirsa VK (2008b) Noise during rest enables the exploration of the brain's dynamic repertoire. PLoS computational biology 4:e1000196.

Glerean E, Salmi J, Lahnakoski JM, Jaaskelainen IP, Sams M (2012) Functional magnetic resonance imaging phase synchronization as a measure of dynamic functional connectivity. Brain connectivity 2:91-101.

Gollo LL, Breakspear M (2014) The frustrated brain: from dynamics on motifs to communities and networks. Philosophical transactions of the Royal Society of London Series B, Biological sciences 369.

Gollo LL, Mirasso C, Sporns O, Breakspear M (2014) Mechanisms of zero-lag synchronization in cortical motifs. PLoS computational biology 10:e1003548.

Gollo LL, Roberts J, Cocchi L (2016) Mapping how local perturbations influence systems-level brain dynamics. Arxiv eprint arXiv:1609.00491.

Goni J, van den Heuvel MP, Avena-Koenigsberger A, Velez de Mendizabal N, Betzel RF, Griffa A, Hagmann P, Corominas-Murtra B, Thiran JP, Sporns O (2014) Resting-brain functional connectivity predicted by analytic measures of network communication. Proceedings of the National Academy of Sciences of the United States of America 111:833-838.

Greicius MD, Supekar K, Menon V, Dougherty RF (2009) Resting-state functional connectivity reflects structural connectivity in the default mode network. Cerebral cortex 19:72-78.

Hagmann P, Cammoun L, Gigandet X, Meuli R, Honey CJ, Wedeen VJ, Sporns O (2008) Mapping the structural core of human cerebral cortex. PLoS biology 6:e159.

Hagmann P, Sporns O, Madan N, Cammoun L, Pienaar R, Wedeen VJ, Meuli R, Thiran JP, Grant PE (2010) White matter maturation reshapes structural connectivity in the late developing human brain. Proceedings of the National Academy of Sciences of the United States of America 107:19067-19072.

Handwerker DA, Roopchansingh V, Gonzalez-Castillo J, Bandettini PA (2012) Periodic changes in fMRI connectivity. NeuroImage 63:1712-1719.

Hansen EC, Battaglia D, Spiegler A, Deco G, Jirsa VK (2015) Functional connectivity dynamics: modeling the switching behavior of the resting state. NeuroImage 105:525-535.

He BJ, Snyder AZ, Zempel JM, Smyth MD, Raichle ME (2008) Electrophysiological correlates of the brain's intrinsic large-scale functional architecture. Proceedings of the National Academy of Sciences of the United States of America 105:16039-16044.

Hermundstad AM, Bassett DS, Brown KS, Aminoff EM, Clewett D, Freeman S, Frithsen A, Johnson A, Tipper CM, Miller MB, Grafton ST, Carlson JM (2013) Structural foundations of resting-state and task-based functional connectivity in the human brain. Proceedings of the National Academy of Sciences of the United States of America 110:6169-6174.

Hindriks R, Adhikari MH, Murayama Y, Ganzetti M, Mantini D, Logothetis NK, Deco G (2015a) Can sliding-window correlations reveal dynamic functional connectivity in resting-state fMRI? NeuroImage 127:242-256.

Hindriks R, Woolrich M, Luckhoo H, Joensson M, Mohseni H, Kringelbach ML, Deco G (2015b) Role of white-matter pathways in coordinating alpha oscillations in resting visual cortex. NeuroImage 106:328-339. 
Hipp JF, Hawellek DJ, Corbetta M, Siegel M, Engel AK (2012) Large-scale cortical correlation structure of spontaneous oscillatory activity. Nature neuroscience 15:884-890.

Hipp JF, Siegel M (2015) BOLD fMRI Correlation Reflects Frequency-Specific Neuronal Correlation. Current biology : CB 25:1368-1374.

Hodgkin AL, Huxley AF (1952) A quantitative description of membrane current and its application to conduction and excitation in nerve. The Journal of physiology 117:500-544.

Honey CJ, Kotter R, Breakspear M, Sporns O (2007) Network structure of cerebral cortex shapes functional connectivity on multiple time scales. Proceedings of the National Academy of Sciences of the United States of America 104:10240-10245.

Honey CJ, Sporns O, Cammoun L, Gigandet X, Thiran JP, Meuli R, Hagmann P (2009) Predicting human resting-state functional connectivity from structural connectivity. Proceedings of the National Academy of Sciences of the United States of America 106:2035-2040.

Hutchison RM, Womelsdorf T, Allen EA, Bandettini PA, Calhoun VD, Corbetta M, Della Penna S, Duyn JH, Glover GH, Gonzalez-Castillo J, Handwerker DA, Keilholz S, Kiviniemi V, Leopold DA, de Pasquale F, Sporns O, Walter M, Chang C (2013a) Dynamic functional connectivity: promise, issues, and interpretations. NeuroImage 80:360-378.

Hutchison RM, Womelsdorf T, Gati JS, Everling S, Menon RS (2013b) Resting-state networks show dynamic functional connectivity in awake humans and anesthetized macaques. Human brain mapping 34:2154-2177.

Izhikevich EM, Edelman GM (2008) Large-scale model of mammalian thalamocortical systems. Proceedings of the National Academy of Sciences of the United States of America 105:3593-3598.

Jones DT, Vemuri P, Murphy MC, Gunter JL, Senjem ML, Machulda MM, Przybelski SA, Gregg BE, Kantarci K, Knopman DS, Boeve BF, Petersen RC, Jack CR, Jr. (2012) Non-stationarity in the "resting brain's" modular architecture. PloS one 7:e39731.

Keller CJ, Bickel S, Honey CJ, Groppe DM, Entz L, Craddock RC, Lado FA, Kelly C, Milham M, Mehta AD (2013) Neurophysiological investigation of spontaneous correlated and anticorrelated fluctuations of the BOLD signal. The Journal of neuroscience : the official journal of the Society for Neuroscience 33:6333-6342.

Laumann TO, Snyder AZ, Mitra A, Gordon EM, Gratton C, Adeyemo B, Gilmore AW, Nelson SM, Berg JJ, Greene DJ, McCarthy JE, Tagliazucchi E, Laufs H, Schlaggar BL, Dosenbach NU, Petersen SE (2016) On the Stability of BOLD fMRI Correlations. Cerebral cortex.

Leonardi N, Shirer WR, Greicius MD, Van De Ville D (2014) Disentangling dynamic networks: Separated and joint expressions of functional connectivity patterns in time. Human brain mapping 35:5984-5995.

Leonardi N, Van De Ville D (2015) On spurious and real fluctuations of dynamic functional connectivity during rest. NeuroImage 104:430-436.

Leopold DA, Murayama Y, Logothetis NK (2003) Very slow activity fluctuations in monkey visual cortex: implications for functional brain imaging. Cerebral cortex 13:422-433. 
Liu X, Duyn JH (2013) Time-varying functional network information extracted from brief instances of spontaneous brain activity. Proceedings of the National Academy of Sciences of the United States of America 110:4392-4397.

Logothetis NK, Pauls J, Augath M, Trinath T, Oeltermann A (2001) Neurophysiological investigation of the basis of the fMRI signal. Nature 412:150-157.

Lowe MJ, Mock BJ, Sorenson JA (1998) Functional connectivity in single and multislice echoplanar imaging using resting-state fluctuations. NeuroImage 7:119-132.

Magri C, Schridde U, Murayama Y, Panzeri S, Logothetis NK (2012) The amplitude and timing of the BOLD signal reflects the relationship between local field potential power at different frequencies. The Journal of neuroscience : the official journal of the Society for Neuroscience 32:1395-1407.

Marzetti L, Della Penna S, Snyder AZ, Pizzella V, Nolte G, de Pasquale F, Romani GL, Corbetta M (2013) Frequency specific interactions of MEG resting state activity within and across brain networks as revealed by the multivariate interaction measure. NeuroImage 79:172-183.

Messe A, Rudrauf D, Benali H, Marrelec G (2014) Relating structure and function in the human brain: relative contributions of anatomy, stationary dynamics, and non-stationarities. PLoS computational biology 10:e1003530.

Messe A, Rudrauf D, Giron A, Marrelec G (2015) Predicting functional connectivity from structural connectivity via computational models using MRI: an extensive comparison study. NeuroImage 111:65-75.

Nakagawa TT, Woolrich M, Luckhoo H, Joensson M, Mohseni H, Kringelbach ML, Jirsa V, Deco G (2014) How delays matter in an oscillatory whole-brain spiking-neuron network model for MEG alpha-rhythms at rest. NeuroImage 87:383-394.

Niebur E, Schuster HG, Kammen DM (1991) Collective frequencies and metastability in networks of limit-cycle oscillators with time delay. Physical review letters 67:2753-2756.

Nir Y, Fisch L, Mukamel R, Gelbard-Sagiv H, Arieli A, Fried I, Malach R (2007) Coupling between neuronal firing rate, gamma LFP, and BOLD fMRI is related to interneuronal correlations. Current biology : CB 17:1275-1285.

Petkoski S, Jirsa V Effects of time-delays for the non-stationary dynamic of the resting state. Clinical Neurophysiology 127:e48.

Ponce-Alvarez A, Deco G, Hagmann P, Romani GL, Mantini D, Corbetta M (2015) Resting-state temporal synchronization networks emerge from connectivity topology and heterogeneity. PLoS computational biology 11:e1004100.

Preti MG, Bolton TA, Ville DV (2016) The dynamic functional connectome: State-ofthe-art and perspectives. NeuroImage.

Robinson PA, Zhao X, Aquino KM, Griffiths JD, Sarkar S, Mehta-Pandejee G (2016) Eigenmodes of brain activity: Neural field theory predictions and comparison with experiment. NeuroImage 142:79-98.

Sakoglu U, Pearlson GD, Kiehl KA, Wang YM, Michael AM, Calhoun VD (2010) A method for evaluating dynamic functional network connectivity and taskmodulation: application to schizophrenia. Magma 23:351-366.

Scholvinck ML, Maier A, Ye FQ, Duyn JH, Leopold DA (2010) Neural basis of global resting-state fMRI activity. Proceedings of the National Academy of Sciences of the United States of America 107:10238-10243. 
Senden M, Goebel R, Deco G (2012) Structural connectivity allows for multithreading during rest: the structure of the cortex leads to efficient alternation between resting state exploratory behavior and default mode processing. NeuroImage 60:2274-2284.

Shanahan M (2010) Metastable chimera states in community-structured oscillator networks. Chaos 20:013108.

Shine JM, Koyejo O, Bell PT, Gorgolewski KJ, Gilat M, Poldrack RA (2015) Estimation of dynamic functional connectivity using Multiplication of Temporal Derivatives. NeuroImage 122:399-407.

Smith SM, Miller KL, Moeller S, Xu J, Auerbach EJ, Woolrich MW, Beckmann CF, Jenkinson M, Andersson J, Glasser MF, Van Essen DC, Feinberg DA, Yacoub ES, Ugurbil K (2012) Temporally-independent functional modes of spontaneous brain activity. Proceedings of the National Academy of Sciences of the United States of America 109:3131-3136.

Spiegler A, Hansen EC, Bernard C, McIntosh AR, Jirsa VK (2016) Selective Activation of Resting-State Networks following Focal Stimulation in a Connectome-Based Network Model of the Human Brain. eNeuro 3.

Sporns O (2011) Networks of the brain. Cambridge, Mass. ; London: MIT Press.

Sporns O, Tononi G, Kotter R (2005) The human connectome: A structural description of the human brain. PLoS computational biology 1:e42.

Stewart I (1999) Mathematics: Holes and hot spots. Nature 401:863-865.

Tagliazucchi E, Balenzuela P, Fraiman D, Chialvo DR (2012a) Criticality in largescale brain FMRI dynamics unveiled by a novel point process analysis. Frontiers in physiology 3:15.

Tagliazucchi E, von Wegner F, Morzelewski A, Brodbeck V, Laufs H (2012b) Dynamic BOLD functional connectivity in humans and its electrophysiological correlates. Frontiers in human neuroscience 6:339.

Takeuchi H, Sekiguchi A, Taki Y, Yokoyama S, Yomogida Y, Komuro N, Yamanouchi T, Suzuki S, Kawashima R (2010) Training of working memory impacts structural connectivity. The Journal of neuroscience : the official journal of the Society for Neuroscience 30:3297-3303.

Tognoli E, Kelso JA (2014) The metastable brain. Neuron 81:35-48.

van den Heuvel MP, Hulshoff Pol HE (2010) Exploring the brain network: a review on resting-state fMRI functional connectivity. European neuropsychopharmacology : the journal of the European College of Neuropsychopharmacology 20:519-534.

van den Heuvel MP, Mandl RC, Kahn RS, Hulshoff Pol HE (2009) Functionally linked resting-state networks reflect the underlying structural connectivity architecture of the human brain. Human brain mapping 30:3127-3141.

Vasa F, Shanahan M, Hellyer PJ, Scott G, Cabral J, Leech R (2015) Effects of lesions on synchrony and metastability in cortical networks. NeuroImage 118:456467.

Vidaurre D, Quinn AJ, Baker AP, Dupret D, Tejero-Cantero A, Woolrich MW (2016) Spectrally resolved fast transient brain states in electrophysiological data. NeuroImage 126:81-95.

Vuksanovic V, Hovel P (2015) Dynamic changes in network synchrony reveal resting-state functional networks. Chaos 25:023116.

Wildie M, Shanahan M (2012) Metastability and chimera states in modular delay and pulse-coupled oscillator networks. Chaos 22:043131. 
Yaesoubi M, Allen EA, Miller RL, Calhoun VD (2015) Dynamic coherence analysis of resting fMRI data to jointly capture state-based phase, frequency, and timedomain information. NeuroImage 120:133-142.

Zalesky A, Breakspear M (2015) Towards a statistical test for functional connectivity dynamics. NeuroImage 114:466-470.

Zalesky A, Fornito A, Cocchi L, Gollo LL, Breakspear M (2014) Time-resolved resting-state brain networks. Proceedings of the National Academy of Sciences of the United States of America 111:10341-10346. 